ARTICLE

\title{
Large-scale computational discovery and analysis of virus-derived microbial nanocompartments
}

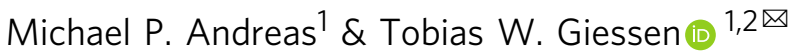

Encapsulins are a class of microbial protein compartments defined by the viral HK97-fold of their capsid protein, self-assembly into icosahedral shells, and dedicated cargo loading mechanism for sequestering specific enzymes. Encapsulins are often misannotated and traditional sequence-based searches yield many false positive hits in the form of phage capsids. Here, we develop an integrated search strategy to carry out a large-scale computational analysis of prokaryotic genomes with the goal of discovering an exhaustive and curated set of all HK97-fold encapsulin-like systems. We find over 6,000 encapsulin-like systems in 31 bacterial and four archaeal phyla, including two novel encapsulin families. We formulate hypotheses about their potential biological functions and biomedical relevance, which range from natural product biosynthesis and stress resistance to carbon metabolism and anaerobic hydrogen production. An evolutionary analysis of encapsulins and related HK97-type virus families shows that they share a common ancestor, and we conclude that encapsulins likely evolved from HK97-type bacteriophages.

\footnotetext{
${ }^{1}$ Department of Biomedical Engineering, University of Michigan Medical School, Ann Arbor, MI, USA. ${ }^{2}$ Department of Biological Chemistry, University of

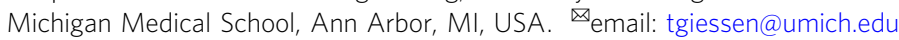


S patial compartmentalization is a ubiquitous feature of biological systems ${ }^{1}$. In fact, biological entities like cells and viruses only exist because of the presence of a barrier that separates their interior from the environment. This concept of creating distinct spaces separate from their surroundings extends further to intracellular organization with many layers of subcompartmentalization found within most cells ${ }^{2,3}$. Intracellular compartments with a proteomically defined interior and a discrete boundary that fulfill distinct biochemical or physiological functions are generally referred to as organelles ${ }^{4}$. This includes both lipid-bound organelles, phase-separated structures, and protein-based compartments. Distinguishing features between eukaryotic lipid-based and prokaryotic protein-based organelles include their size range--micro vs. nano scale--and the fact that protein organelle structure is genetically encoded and thus generally more defined. Still, compartmentalization, however it is achieved, can ultimately serve four distinct functions, namely, the creation of distinct reaction spaces and environments, storage, transport, and regulation ${ }^{4}$. Often, compartmentalization can serve multiple of these functions at the same time. More specifically, the functions of intracellular compartments include sequestering toxic reactions and metabolites, creating distinct biochemical environments to stimulate enzyme or pathway activity, and dynamically storing nutrients for later use, among many others ${ }^{4}$.

One of the most widespread and diverse classes of proteinbased compartments are encapsulin nanocompartments, or simply encapsulins ${ }^{5-7}$. So far, two families of encapsulins have been reported in a variety of bacterial and archaeal phyla ${ }^{8-10}$. They are proposed to be involved in oxidative stress resistance ${ }^{9,11-13}$, iron mineralization and storage ${ }^{14,15}$, anaerobic ammonium oxidation $^{16}$, and sulfur metabolism ${ }^{8}$. All experimentally studied encapsulins self-assemble from a single capsid protein into compartments between 24 and $42 \mathrm{~nm}$ in diameter with either $T=1, T=3$ or $T=4$ icosahedral symmetry ${ }^{10,12,15}$. Their defining feature is the ability to selectively encapsulate cargo proteins which include ferritin-like proteins, hemerythrins, peroxidases and desulfurases ${ }^{8,9}$. In classical encapsulins (Family 1), encapsulation is mediated by short C-terminal peptide sequences referred to as targeting peptides (TPs) or cargo-loading peptides (CLPs) $^{10,15,17}$ while for Family 2 systems, larger N-terminal protein domains are proposed to mediate encapsulation ${ }^{8}$. Nterminal TPs have also been observed in secondary cargo ferredoxin proteins from Firmicutes encapsulin systems 9 . For most encapsulin systems, little is known about the specific reasons or functional consequences of enzyme encapsulation. Suggestions include the sequestration of toxic or reactive intermediates as well as enhancing enzyme activity and the prevention of unwanted side reactions. Many organisms encode multiple distinct encapsulin systems which likely sequester different cargo proteins. Neighboring encapsulin-encoding genes have recently been identified in the same operon, opening the possibility for heteromeric mixed capsids. One of the most intriguing features of encapsulins is that in contrast to all other known protein-based compartments or organelles, their capsid monomer shares the HK97 phage-like fold ${ }^{10,12,15}$. This has led to the suggestion that encapsulins are derived from or in some way connected to the world of phages and viruses ${ }^{5,9}$.

Here, we carry out a large-scale in-depth computational analysis of prokaryotic genomes with the goal of discovering and classifying an exhaustive set of all HK97-type protein organelle systems. We develop a Hidden Markov Model (HMM)-, Pfam family-, and genome neighborhood analysis (GNA)-based search strategy and substantially expand the number of identified encapsulin-like operons. We report the discovery and analysis of two novel encapsulin families (Family 3 and Family 4 ) as well as many new operon types that fall within Family 1 and Family 2. We formulate data-driven hypotheses about the potential biological functions of newly identified operons which will guide future experimental studies of encapsulin-like systems. Further, we conduct a detailed evolutionary analysis of encapsulin-like systems and related HK97-type virus families and show that encapsulins and HK97-type viruses share a common ancestor and that encapsulins likely evolved from HK97-type phages. Our study sheds new light on the evolutionary interplay of viruses and cellular organisms, the recruitment of protein folds for novel functions, and the functional diversity of microbial protein organelles.

\section{Results and discussion}

Distribution, diversity, and classification of encapsulin systems found in prokaryotes. All bacterial and archaeal proteomes available in the UniProtKB ${ }^{18}$ database (Family 1, 2, and 4: March 2020; Family 3: February 2021) were analyzed for the presence of encapsulin-like proteins using an HMM-based search strategy. It was discovered that all Pfam families associated with initial search hits belong to a single Pfam clan (CL0373) ${ }^{19}$ encompassing the majority of HK97-fold proteins catalogued in the Pfam database. Thus, we supplemented our initial hit dataset with all sequences associated with CL0373. This was followed by GNA-based curation ${ }^{20}$ of the expanded dataset to remove all false.

Positives, primarily phage genomes, resulting in a curated list of 6,133 encapsulin-like proteins (Fig. 1 and Supplementary Data 1). Encapsulin-like systems can be found in 31 bacterial and 4 archaeal phyla. Based on the sequence similarity and Pfam family membership of identified capsid proteins, and the genomeneighborhood composition of associated operons, encapsulin-like systems could be classified into 4 distinct families (Fig. 2). Family 1 and 2 represent previously identified encapsulin operon types containing capsid proteins falsely annotated as bacteriocin (PF04454: Linocin_M18) and transcriptional regulator/membrane protein (no Pfam), respectively. Family 1 will be referred to as Classical Encapsulins given the fact that they were the first discovered and are the best characterized. Family 3 and 4 represent newly discovered systems. Family 3 encapsulins are falsely annotated as phage major capsid protein (PF05065: Phage_capsid) and are found embedded within large biosynthetic gene clusters (BGCs) encoding the biosynthetic machinery for different peptide-based natural products. Therefore, Family 3 was dubbed Natural Product Encapsulins. Family 4 is characterized by a highly truncated encapsulin-like capsid protein which is generally annotated as an uncharacterized protein (PF08967: DUF1884) and arranged in conserved two-component operons with different enzymes. Family 4 proteins represent the Adomain of the canonical HK97-fold with all other domains usually associated with this fold missing. Thus, Family 4 will be referred to as A-domain Encapsulins.

Classical Encapsulins (Family 1) represent the most widespread family of encapsulin-like systems. They can be found in 31 out of 35 prokaryotic phyla found to encode encapsulin-like operons (Fig. 1). 2,383 Classical Encapsulin operons were discovered with the phyla Proteobacteria, Actinobacteria and Firmicutes containing the majority of identified systems. However, it should be noted that these phyla.

Also contain the largest number of sequenced genomes and available proteomes. Family 1 contains at least 6 operon types defined by the presence of 6 distinct and conserved cargo 


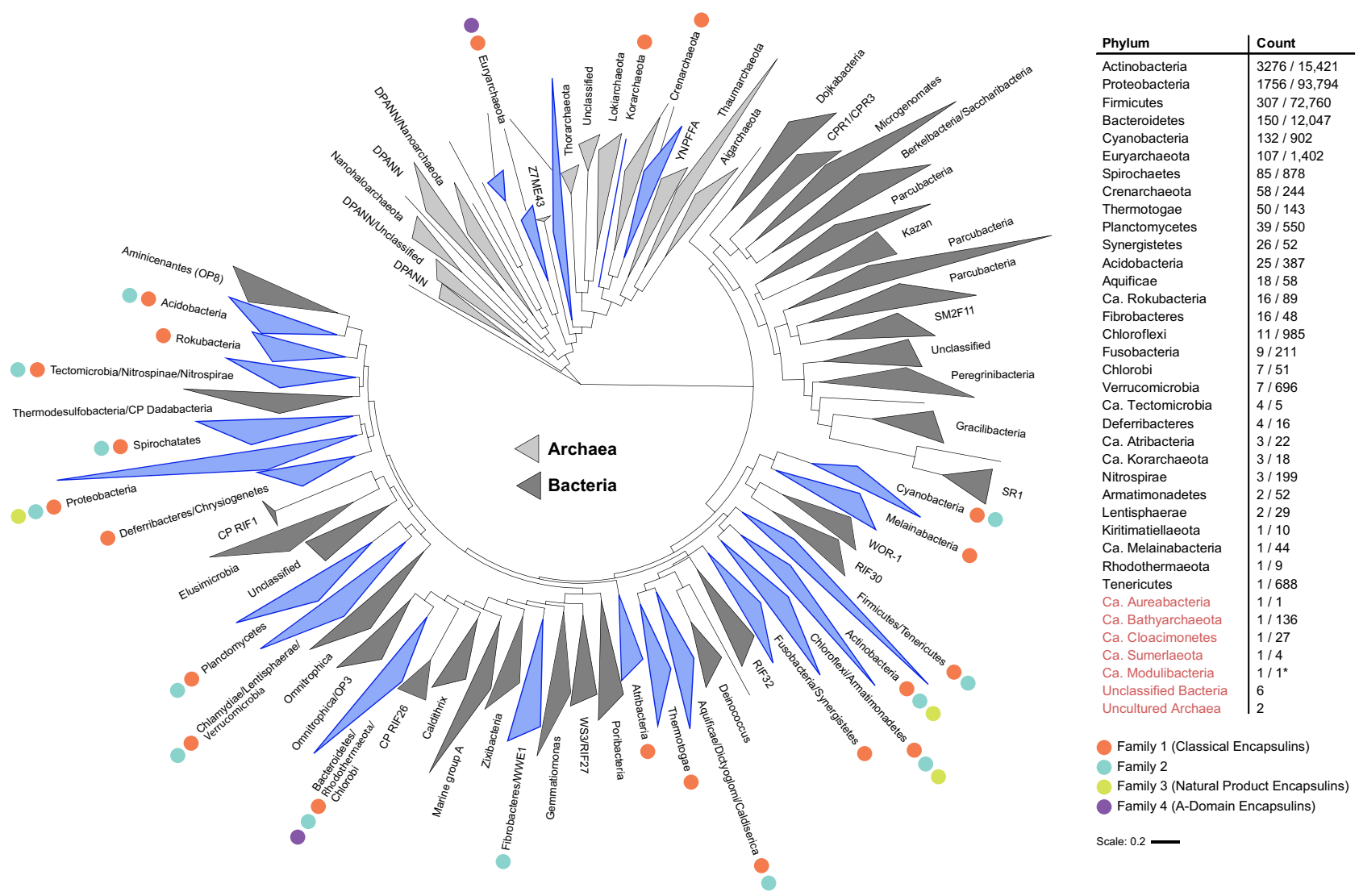

Fig. 1 Distribution of encapsulin-like systems in prokaryotes. Left: Phylogenetic tree based on 108 of the major archaeal and bacterial phyla118. Phyla containing encapsulin-like systems are highlighted in blue. Differently colored dots indicate the presence of the respective encapsulin family within the phylum. Right: List of phyla discovered to encode encapsulin-like systems. The Count column shows the number of identified systems and the total number of proteomes available in UniProt (\# systems identified/\# UniProt proteomes). Ca. refers to candidate phyla. Phylum names colored red show new phyla or uncultured/unclassified organisms not shown in the phylogenetic tree. ${ }^{\star} \mathrm{Ca}$. Modulibacteria is not an annotated phylum in UniProt but has been proposed as a candidate phylum ${ }^{131}$.

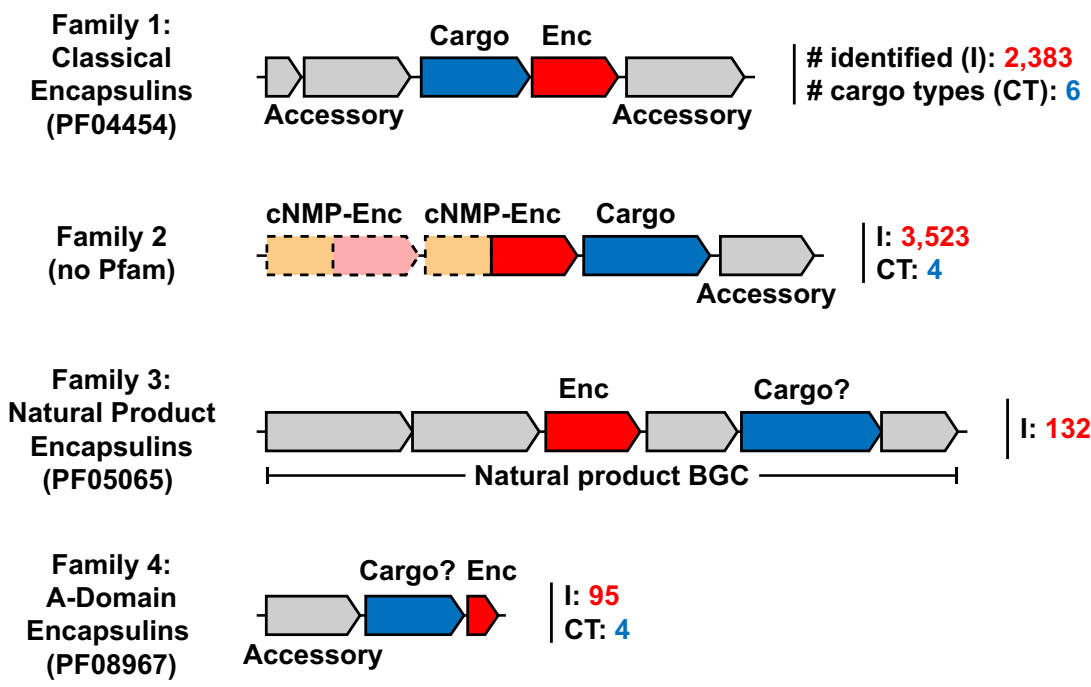

Fig. 2 Novel classification scheme for encapsulin-like operons. Shown are the four newly defined families of encapsulins with the respective Pfam annotations if available. Encapsulin-like capsid components are shown in red. Confirmed and proposed cargo proteins are shown in blue. Non-cargo accessory components are shown in grey. The number of identified systems of a given family is shown after the operon in red (I, \# identified) and the number of distinct cargo types is shown in cyan (CT, \# cargo types). Dotted lines indicate optional presence of operon components. CNMP: cyclic nucleotide-binding domain (orange), Enc: encapsulin-like capsid component. BGC biosynthetic gene cluster. 
proteins. Many of these operon types can be found in distantly related phyla consistent with frequent horizontal gene transfer events. The general operon organization of Family 1 systems consists of the encapsulin capsid protein and a single primary cargo protein usually encoded directly upstream of the shell component (Fig. 2) . Depending on the operon type, other conserved accessory components can be present ${ }^{9,15}$. These components are not cargo proteins but are proposed to be directly involved in the biochemical function or regulation of a given system.

Family 2 encapsulins are the most numerous encapsulin-like systems and can be found in 14 bacterial phyla (Fig. 1). 3,523 Family 2 operons were identified. The majority of systems can be found in the phyla Actinobacteria and Proteobacteria followed by Bacteroidetes and Cyanobacteria. Family 2 contains at least four different operon types based on cargo protein identity. Again, the widespread occurrence of these operon types in distant phyla supports the hypothesis of their frequent horizontal transfer. Family 2 operon organization is more complex compared to Family 1 due to the variable presence of a cNMP-binding domain (PF00027) fused to the encapsulin capsid component as well as the variable occurrence of two distinct capsid components within a single Family 2 operon. Further non-cargo accessory components may be present, likely related to the biological function of a given operon (Fig. 2) ${ }^{8}$.

Natural Product Encapsulins (Family 3) can be found almost exclusively in the phyla Actinobacteria and Proteobacteria, primarily in Streptomyces and Myxococcus species as well as some other closely related genera (Fig. 1). Streptomyces and Myxococcus species are widely known as being among the most prolific producers of bioactive natural products ${ }^{21,22}$. So far, 132 Family 3 systems have been identified and can be classified into 6 distinct operon types based on the organization of the BGC surrounding the encapsulin capsid component (Fig. 2).

A-domain Encapsulins (Family 4) are the most distinct family so far discovered and are restricted to the archaeal phylum Euryarchaeota and the bacterial phylum Bacteroidetes (Fig. 1). 95 Family 4 operons have been identified with more than 90 percent found in Archaea. Family 4 encapsulin-like proteins are truncated to around 100 amino acids in length and thus only one third the length of a standard HK97-fold protein ${ }^{23,24}$. All archaeal Family 4 operons consist of a single- or multi-subunit enzyme and the Adomain Encapsulin protein located downstream of the enzymatic component (Fig. 2). Some systems seem to possess further accessory components as part of the operon as judged by overlapping genes and transcription direction. So far, four distinct archaeal operon types have been discovered. The identified bacterial A-domain Encapsulins are not arranged in an obvious operon-like structure which makes their classification and function prediction more difficult.

Family 1 - Classical encapsulins. Our dataset of 2,383 Family 1 systems greatly expands the set of the previously described 932 Classical Encapsulins (Fig. 3) ${ }^{9,16}$. 1,505 dye-decolorizing peroxidase (DyP) systems were identified, making them the most abundant cargo class in Family 1. DyP systems are most abundant in Actinobacteria and Proteobacteria, with 962 and 519 systems found in each phylum, respectively. DyP peroxidases bind heme and are named for their ability to oxidize a broad range of anthraquinone dyes ${ }^{25}$. DyPs have also been shown to break down lignin and other typical peroxidase substrates ${ }^{26,27}$.

Encapsulated DyP from Brevibacterium linens has been shown to form a trimer of dimers with D3 symmetry and bind close to the three-fold symmetry axis of the encapsulin shell via C-terminal targeting peptides ${ }^{10}$. A more recent cryo-EM reconstruction of encapsulated DyP from Mycobacterium smegmatis however, shows two DyP hexamers arranged with D2 symmetry, indicating variability in the arrangement of encapsulated DyP multimers ${ }^{28}$. Many DyP Family 1 operons in Mycobacteria contain accessory genes encoding short chain oxidoreductases and cupins in addition to the core DyP cargo. Their function within the context of DyP encapsulin operons is currently unknown (Fig. S1a). Accessory genes encoding putative membrane proteins containing DUF 1345 domains are commonly found in DyP-containing operons in Streptomyces and might play a role in transport related to DyP function (Fig. S1a). Further, 67 DyP operons were identified in Streptomyces that contain accessory genes encoding for a DUF5709 domain protein and genes annotated as 6-phosphogluconate dehydrogenases (6-GPD) and diaminopimelate decarboxylases (DAPDC) (Fig. S1a). Both 6-GPD and DAPDC possess decarboxylase activity and play key roles in the pentose phosphate pathway and amino acid biosynthesis, however, their role in the context of DyP encapsulin systems is currently unknown. The general biological function of DyP encapsulin systems is still speculative, however, a recent study showed that a DyP Family 1 system in Mycobacterium tuberculosis plays a direct role in oxidative stress resistance during infection ${ }^{11}$.

Ferritin-like proteins (FLPs) comprise the second largest set of cargo proteins associated with Family 1 encapsulins. FLPs represent a large functionally diverse superfamily of proteins that all share a four-helix bundle fold. Clustering encapsulinassociated FLPs at 30\% sequence identity results in 7 distinct families that largely correspond to the following Pfam families: Flp (lower case to distinguish the Pfam family from the superfamily), rubrerythrin, CoQ7, Mn catalase, IMEF, hemerythrin, and bacterioferritin (Fig. S2).

Identified Flp, rubrerythrin, CoQ7, and $\mathrm{Mn}$ catalase cargo proteins are likely functionally identical--acting as ferroxidases--and should likely be part of the same Pfam family. From now on, we will refer to all four simply as Flp cargos. They are found in 23 bacterial and 2 archaeal phyla. They are widespread in bacteria but predominantly found in Firmicutes and Proteobacteria. Crystal structures of encapsulin-associated Flps from Haliangium ochraceum and Rhodospirillum rubrum suggest that these systems form decameric assemblies with D5 symmetry (Fig. S2) ${ }^{14,29}$. Recent cryo-EM reconstructions of the T. maritima encapsulin demonstrate that up to five decameric Flp complexes can be encapsulated, with the most common encapsulin-cargo complex exhibiting four Flp decamers arranged with tetrahedral-like symmetry ${ }^{30}$. Unlike ferritin cages with higher symmetries, Flp cargo proteins cannot store precipitated iron in a soluble form by themselves and rely on the encapsulin shell to achieve iron precipitate sequestration. Similar to the ubiquitous ferritin iron storage cages, Flp encapsulin systems might play a dual role in oxidative stress resistance and iron homeostasis.

The second-largest cargo class within the FLP superfamily is the iron-mineralizing encapsulin-associated Firmicute (IMEF) cargos. They form dimers in solution and when encapsulated and are most commonly found in Firmicutes. Encapsulins containing these systems are capable of forming large $\mathrm{T}=4$ capsids approximately $42 \mathrm{~nm}$ in diameter ${ }^{9,15}$. The large size of these assemblies allows them to form iron-rich cores up to $30 \mathrm{~nm}$ in diameter, making them the largest protein-based iron storage system known to date. Many IMEF-containing operons encode $2 \mathrm{Fe}-2 \mathrm{~S}$ ferredoxins (Fdxs) homologous to bacterioferritinassociated ferredoxins (Bfds) (Fig. S1). Bfd proteins assist in the mobilization of iron from iron-filled ferritin cages ${ }^{31}$. Many of the identified Fdxs contain a strongly conserved targeting peptidelike TVGSL motif at their N-terminus and have been shown to 


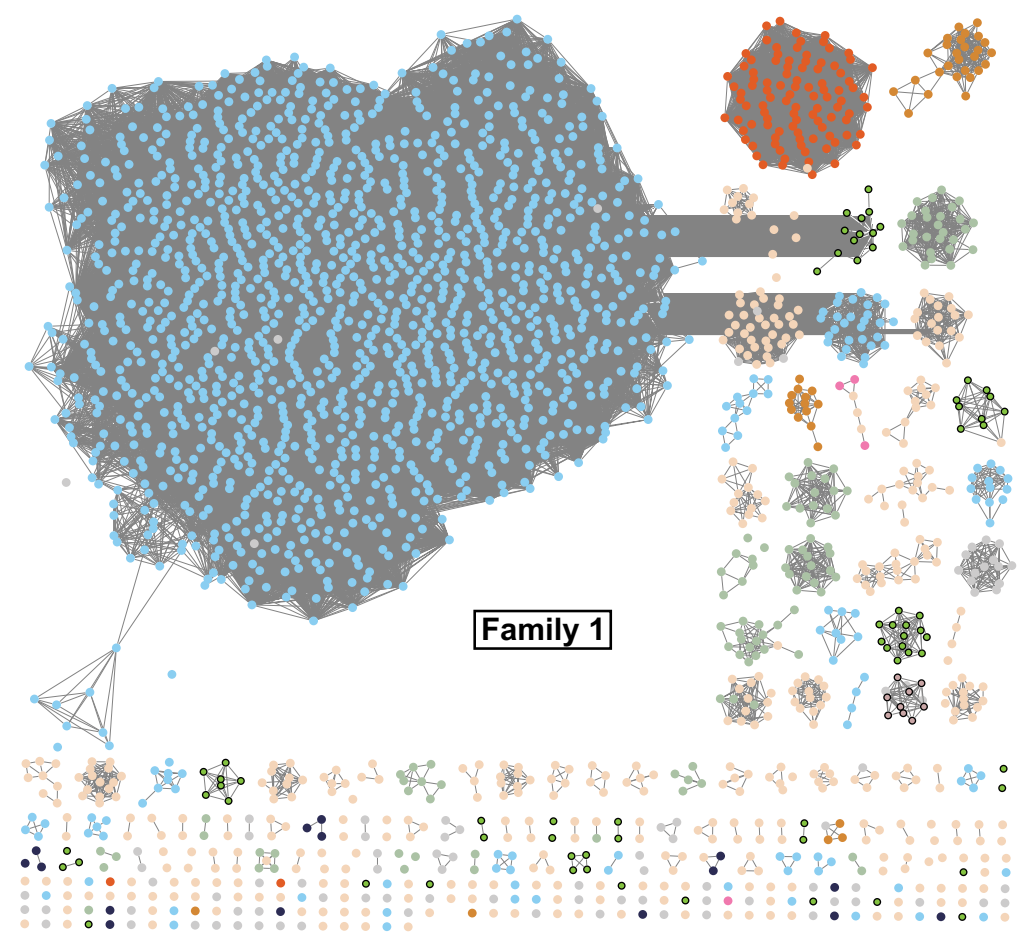

b

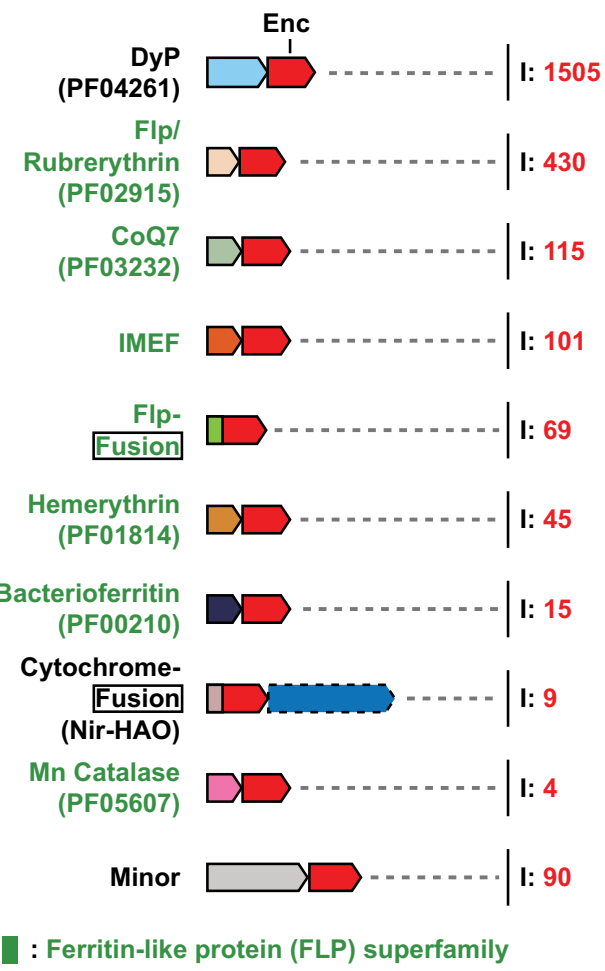

Fig. 3 Overview and analysis of Family 1 encapsulin systems. a SSN analysis of 2383 Family 1 encapsulins clustered at $49 \%$ sequence identity. Nodes are colored based on the associated primary cargo type shown in (b). b Diversity of Family 1 operon types. Only conserved primary cargo proteins are shown. Operons not containing any of the main cargo types are designated as Minor and are shown in more detail in Fig. S1. I number of identified operons.

co-purify with IMEF encapsulins when heterologously expressed ${ }^{9}$. Fdxs might be involved in releasing stored iron from IMEF encapsulins by transferring electrons to the interior of the capsid, thus reducing and solubilizing stored iron. Most organisms encoding IMEF systems do not encode any classical ferritins making it likely that IMEF encapsulins act as their primary iron storage compartments.

Within the FLP superfamily, 45 hemerythrin cargos were identified, with 42 found in Actinobacteria and 3 in Proteobacteria. No hemerythrin-containing encapsulin has been structurally characterized, but hemerythrins have been shown to form dimers in solution ${ }^{9}$. Hemerythrin cargos have further been shown to offer oxidative and nitrosative stress protection when encapsulated $^{9}$. All hemerythrins contain binuclear iron centers which have been shown to bind to nitric oxide, oxygen, and other reactive or volatile small molecules ${ }^{32,33}$. Family 1 hemerythrin systems are thus likely involved in the sequestration and detoxification of harmful compounds.

Another FLP cargo type identified in a small number of Firmicutes, Aquificae, Chlorflexi, and Cyanobacteria are bacterioferritins (Bfrs). These putative cargos are composed of two fourhelix bundles and are thus structurally distinct from the other identified FLP superfamily cargos (Fig. S2). Bfrs generally assemble into 24 subunit $12 \mathrm{~nm}$ cages able to store iron - similar to eukaryotic ferritins ${ }^{34}$. A bacterioferritin (BfrB) encoded outside a Family 1 operon has been proposed to be a potential cargo protein in M. tuberculosis, however, no Family 1 operon encoding a Bfr cargo protein has been reported before ${ }^{13}$. The presence of conserved C-terminal targeting peptides in the identified Bfrs strongly suggests that they are encapsulin cargos. The biological function and underlying logic of a putative shell-within-a-shell arrangement in the context of iron storage compartments is currently unknown.
The number of Flp-fusion encapsulins was also expanded. In these systems, an Flp domain is N-terminally fused to the encapsulin capsid protein. This leads to the internalization of Flp domains upon capsid self-assembly. All Flp-fusion systems are present in Archaea, mostly in the phylum Crenarchaeota. Structural studies of Pyrococcus furiosus and Sulfolobus solfataricus Flp-fusion encapsulins have shown that these systems assemble into $\mathrm{T}=3$ capsids and contain internalized Flp assemblies $^{35,36}$. While the excised $P$. furiosus Flp domain has been shown to form a decamer with D5 symmetry - similar to other characterized Flp cargos - the structural arrangement of fused and encapsulated Flps remains unknown ${ }^{14}$. Flp-fusion encapsulins are often located in operons containing other ferritinlike proteins or rubrerythrins, hinting at a function related to iron homeostasis and stress resistance (Fig. S1).

Another type of Family 1 encapsulin fusion system was identified in Planctomycetes. 9 encapsulin-encoding genes with an $\mathrm{N}$-terminal diheme cytochrome $\mathrm{C}$ fusion domain were identified. All cytochrome-fusion systems are found in anammox bacteria and are associated with a nitrite reductase-hydroxylamine oxidoreductase (NIR-HAO)-encoding gene. These systems have been shown to form $\mathrm{T}=3$ icosahedral compartments ${ }^{9}$. Their biological function is currently unknown, however, a role in detoxifying harmful intermediates of the anammox process like nitric oxide, hydroxylamine, and hydrazine, has been proposed, as well as a role in iron storage inside the anammoxosome--the membrane-bound compartment sequestering the anammox process in anammox bacteria ${ }^{9,16}$. An N-terminal Sec-like signal peptide in this encapsulin may be responsible for targeting it to the anammoxosome interior in an unfolded state, allowing the maturation of the fused cytochrome $\mathrm{C}$ domain during or after membrane translocation, followed by capsid assembly inside the anammoxosome organelle $\mathrm{e}^{37,38}$. 
90 Family 1 systems were identified which could not be assigned to any of the so far discussed operon types. They are present in a broad range of phyla and are found within diverse genome neighborhoods (Fig. S1). Of note are a number of systems found in Streptomyces and Mesorhizobium species with conserved N-terminal truncations of the encapsulin capsid gene which might indicate a divergent mode of capsid assembly (Fig. S1b).

Family 2. Family 2 encapsulins are the most abundant class of encapsulins identified in this study and can be broadly grouped into two structurally distinct variants: Family 2 A and Family $2 \mathrm{~B}$. A classification of Family 2 systems was previously proposed based on phylogeny and cargo protein type ${ }^{8}$. However, with our more expanded dataset, it became clear that a classification based on the most distinctive feature of this class, namely, the absence (2 A) or presence (2B) of an internal cNMP-binding domain, would be more appropriate. All Family 2 encapsulins display the HK97-like fold but do not contain an elongated N-terminal helix seen in Family 1 encapsulins (Fig. S3). Instead, they possess an extended $\mathrm{N}$-arm with a short $\mathrm{N}$-terminal $\alpha$-helix (N-helix), more characteristic of the canonical HK97 fold found in bacteriophages $^{8}$. A single Family $2 \mathrm{~A}$ structure has been solved (Synechococcus elongatus) (PDB: $6 \mathrm{X} 8 \mathrm{M}$ and $6 \mathrm{X} 8 \mathrm{~T})^{8}$ which showed that the $\mathrm{N}$-terminus, including the $\mathrm{N}$-helix, extends towards the outside of the capsid, in contrast to Family 1 encapsulins where the $\mathrm{N}$-terminus is sequestered inside the protein shell. Family 2 encapsulins generally contain an extended $\mathrm{N}$-terminal sequence (N-extension) preceding the $\mathrm{N}$-helix (Fig. S3). Family $2 \mathrm{~A}$ encapsulins display short $\mathrm{N}$-extensions around 11 amino acids long while Family $2 \mathrm{~B}$ encapsulins tend to have longer $\mathrm{N}$-extensions around 18 amino acids in length. All Nextensions are predicted to be disordered. In the extreme case of the Streptomyces parvulus Family 2 system, the $\mathrm{N}$-extension is 88 amino acids long. The putative cNMP-binding domains in Family 2B encapsulins are also highly variable, sharing only $19 \%$ pairwise identity between all identified domains. The cNMP-binding domain is connected to the C-terminal fragment of the E-loop via a poorly conserved ca. 60 amino acid linker that is predicted to be disordered (Fig. S3). The presence of the cNMP-binding domain suggests that Family $2 \mathrm{~B}$ encapsulins may regulate encapsulated components in a cNMP-dependent manner, providing these systems with a novel mode of enzyme regulation via sequestration inside a protein shell, not seen in any other encapsulin family.

The most commonly enriched genes associated with Family 2 encapsulins encode for cysteine desulfurases (CD), terpene cyclases (TC), polyprenyl transferases (PT), and xylulose kinases (XK) (Fig. 4a, b). With the exception of xylulose kinases, all of these genes encode proteins with large unannotated regions at their termini, generally predicted to be disordered, which may be involved in mediating cargo encapsulation (Fig. 4c and Fig. S4). Family 2B operons often encode two distinct cNMP-domaincontaining encapsulin capsid proteins (Fig. 4b). This opens up the intriguing possibility of encapsulins forming two-component shells. Family 2B capsids encoded within the same operon roughly share $60 \%$ sequence identity with the main differences being primarily found in the E-loops and putative cNMP-binding domains. This relatively low sequence identity, the localized sequence differences, and the conservation of double shell systems across many phyla and cargo types likely means that encoding two capsid proteins in a single operon is a feature of these systems and not the result of a recent gene duplication event. The presence of two distinct regulatory cNMP-binding domains within the same capsid may allow the fine-tuning of the activity of encapsulated cargo. However, we can currently not exclude that these operons encode two separately assembling encapsulins instead of a single mixed shell.

The partially characterized S. elongatus Family 2A encapsulin has been shown to encapsulate a CD cargo protein and to be upregulated during sulfur starvation ${ }^{8}$. We have identified 1281 Family 2A and 199 Family 2B encapsulin-encoding operons containing CDs as the putative cargo (Fig. 4). Family 2A CD systems are present in 12 bacterial phyla and are most abundant in Proteobacteria (813), Actinobacteria (193), and Bacteroidetes (111). Family 2B CD systems can be found in 9 phyla with a similar distribution as Family 2A systems. The N-termini of CDs are largely predicted to be disordered and are not annotated while the C-terminal region contains a conserved SufS-like cysteine desulfurase domain (PF00266) (Fig. S4) that usually converts cysteine to alanine whilst using the liberated sulfur atom to form a protein-bound persulfide intermediate which is then transferred to sulfur acceptor proteins ${ }^{39}$. While no specific targeting peptide has been identified in CD systems, the unannotated N-terminal domain has been shown to be responsible for mediating encapsulation ${ }^{8}$. Serine $O$-acetyltransferases and rhodaneses are the most highly enriched accessory components found in these operons (Fig. S5). Serine $O$-acetyltransferases catalyze the formation of $O$-acetyl-serine, which is then converted to cysteine via cysteine synthase. Rhodaneses typically act as sulfur atom acceptors, distributing sulfur to various metabolic pathways and processes including cofactor biosynthesis and iron-sulfur cluster formation $^{40}$. Sequestering a CD inside a protein shell might ensure that only a specific co-regulated rhodanese able to interact with the encapsulin capsid exterior can act as the sulfur acceptor thus making sure that sulfur is channeled to a specific subset of metabolic targets. The presence of these operon components suggests that Family 2A CD systems play a role in sulfur utilization and redox homeostasis.

TC- and PT-encoding genes are highly enriched in many Family 2B operons suggesting a role in terpenoid biosynthesis (Fig. 4). We have identified 904 Family 2B operons encoding TCs as their putative cargo. They are commonly found in Actinobacteria (724), Proteobacteria (114), and Cyanobacteria (64). PT systems were found in 900 operons--almost exclusively in Actinobacteria (888). 165 systems were found to encode both TCs and PTs in the same gene cluster. The operon structure of these systems is highly diverse. Many TC systems encode $C$ methyltransferases, usually associated with 2-methylisoborneolsynthase (2-MIBS)-like TCs. Isopentenyl pyrophosphate isomerases and alcohol dehydrogenases are also enriched in TC operons and likely add to the diversity of terpenoid products produced by these systems (Fig. S5). PT systems often encode genes involved in terpenoid precursor biosynthesis. Other genes enriched in PT operons encode terpenoid tailoring enzymes like epimerases, dehydrogenases, acetyltransferases, and deaminases indicating that PT systems are capable of producing a highly diverse array of terpenoids.

Family 2-associated TCs can be classified into two groups: 2MIBS-like cyclases, and geosmin synthase (GS)-like cyclases (Fig. S6). 2-MIB is a monoterpenoid derivative that is formed from the cyclization of 2-methylgeranyl diphosphate. Geosmin is a diterpenoid resulting from the cyclization of farnesyl diphosphate. Structurally, the 2-MIBS-like cyclases contain a single TC domain near the C-terminal half of the protein while the first 100 to 120 amino acids are usually unannotated and often predicted to be disordered (Fig. 4c and Fig. S4). In contrast, GS-like cyclases contain two TC domains. Sequence alignments show that most 2MIBS-like cyclases contain a conserved glycine, proline, and alanine-rich region within the unannotated N-terminal domain (consensus: GPTGLGT) (Fig. S4). Similarly, GS-like cyclases contain a conserved GPTGLGTSAAR (consensus) sequence 
a
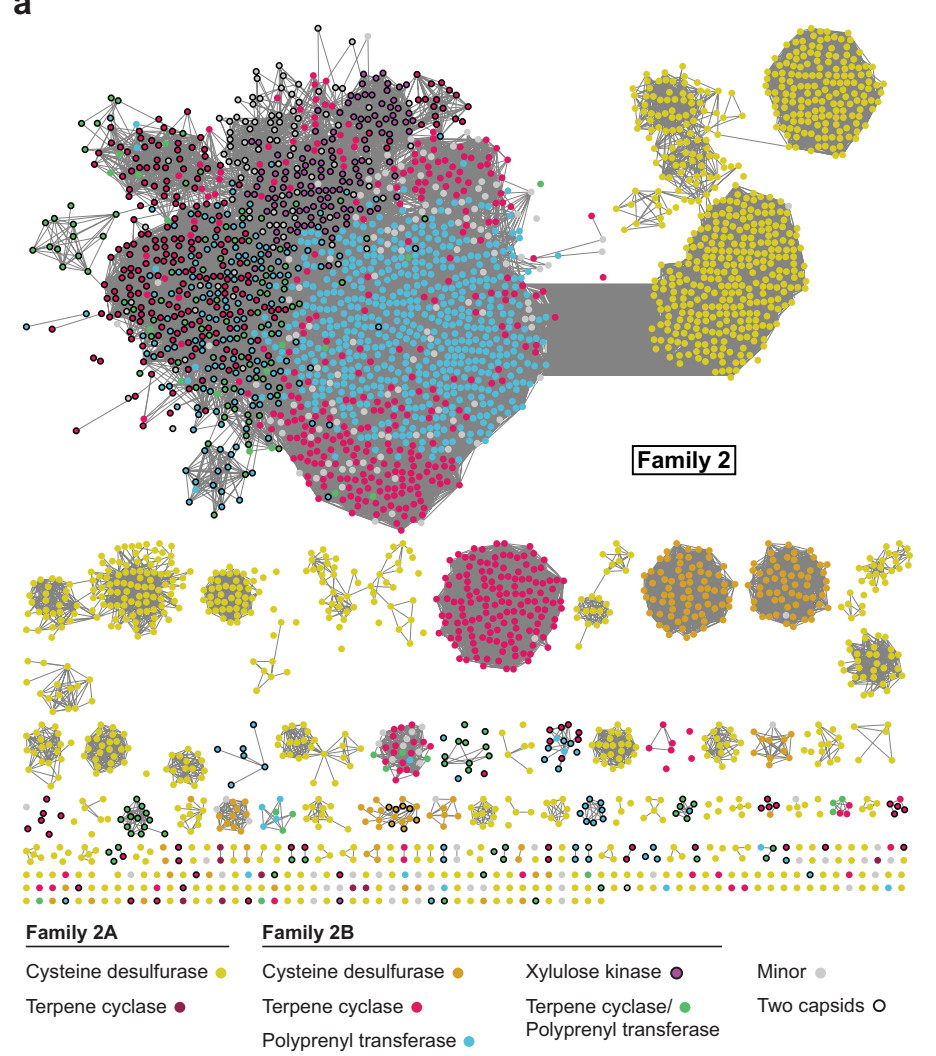
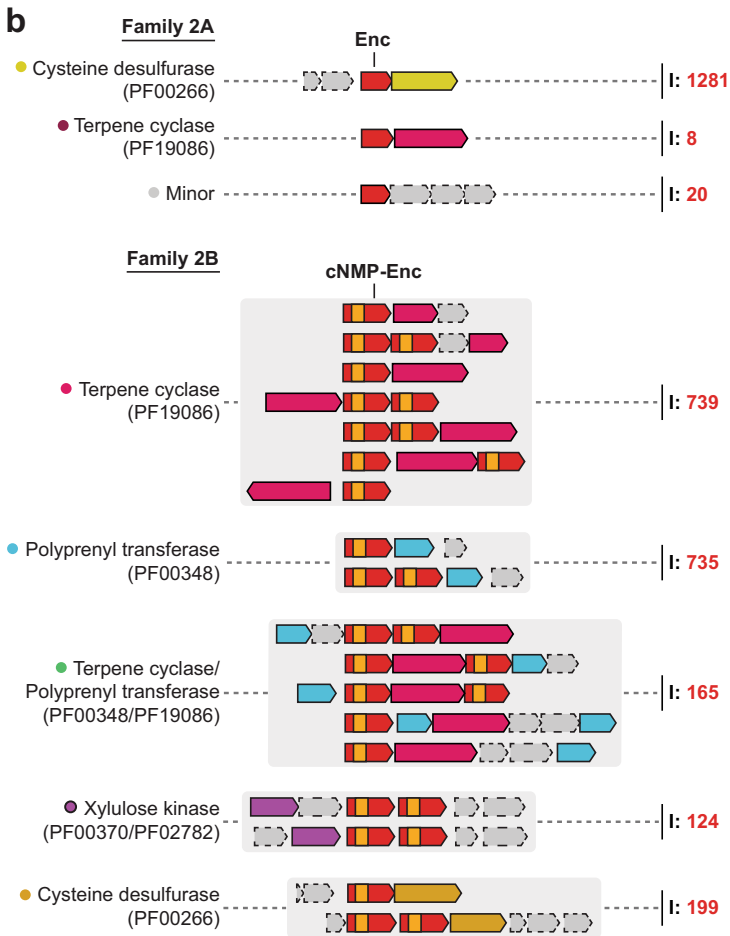

(PF00266)

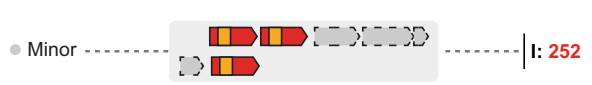

[-.... Variable enriched components

C
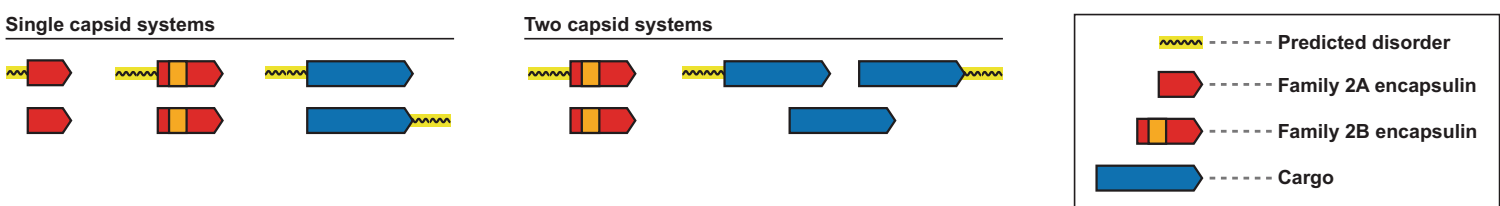

Fig. 4 Overview and analysis of Family 2 encapsulin systems. a SSN analysis of 3523 Family 2 encapsulins clustered at $70 \%$ sequence identity. Nodes are colored based on the putative associated cargo type. Family 2 A: no cNMP domain, Family 2B: cNMP domain present. $\mathbf{b}$ Selection of operon types encoding Family 2 encapsulins. Operons are grouped by their conserved putative cargo protein type. c Combinations of commonly observed extended disordered regions at the termini of Family 2 encapsulins and associated cargo proteins. I: number of identified operons.

between the two cyclase domains which is repeated at the very Cterminus of the protein (Fig. S4). These conserved motifs located in unannotated and disordered regions of TCs may function as targeting sequences responsible for mediating cargo encapsulation.

Family 2B-associated PTs are highly diverse and likely capable of producing linear isoprenoids of varying lengths (Fig. S7). Similar to other Family 2B cargos, encapsulin-associated PTs have a disordered N-terminal domain of 50 to 100 residues (Fig. 4c and Fig. S4). No conserved sequence motifs that may function as targeting tags could be identified. However, the consistent presence of unannotated and disordered domains may suggest their involvement in PT cargo encapsulation.

124 Family 2B systems exclusively found in Streptomyces contained enriched $x y l B$ genes encoding for XKs (Fig. 4). All XK gene clusters contained two distinct Family $2 \mathrm{~B}$ encapsulins indicating that the formation of a putative two-component shell might be essential for these systems. Comparing the sequences between the first and second encapsulins in these XK operons reveals that the HK97-like domains generally share 75\% sequence identity, whereas the putative cNMP-binding domains tend to share around $60 \%$ sequence identity. The high sequence conservation observed between these HK97-like domains may support the abovementioned hypothesis that they may be capable of forming heteromeric shells. In contrast to the other identified Family 2 cargo types, XKs do not consistently contain stretches of predicted disorder or unannotated domains. Commonly enriched accessory components such as acetylxylan esterases, xylose repressors $(x y l R)$, and xylose isomerases $(x y l A)$ suggest that these Family $2 \mathrm{~B}$ systems may be involved in xylose utilization and metabolism (Fig. S5).

Family 3 - Natural product encapsulins. We identified 132 Family 3 encapsulins encoded in a variety of different natural product biosynthetic gene clusters (BGCs) (Fig. 5). 97 Family 3 encapsulins can be found in Actinobacteria, 34 in Proteobacteria, and one in Chloroflexi. We categorized Family 3 encapsulins according to their sequence similarity and surrounding BGC type into 6 classes (Fig. 5b). Classes were named based on the most prominent genera encoding a given class. Family 3 BGCs encode diverse components but commonly found genes include sulfotransferases, short-chain dehydrogenases (SDRs), polyketide synthases (PKSs), non-ribosomal peptide synthetases (NRPSs), and amino-group carrier proteins (AmCPs).

Only one Family 3 encapsulin-containing BGC has been studied experimentally, namely, a system found in Myxococcus sp. 
a

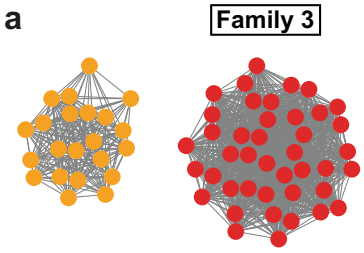

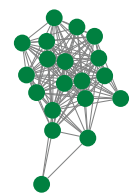
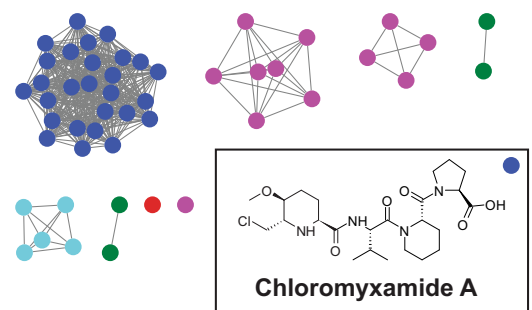

b
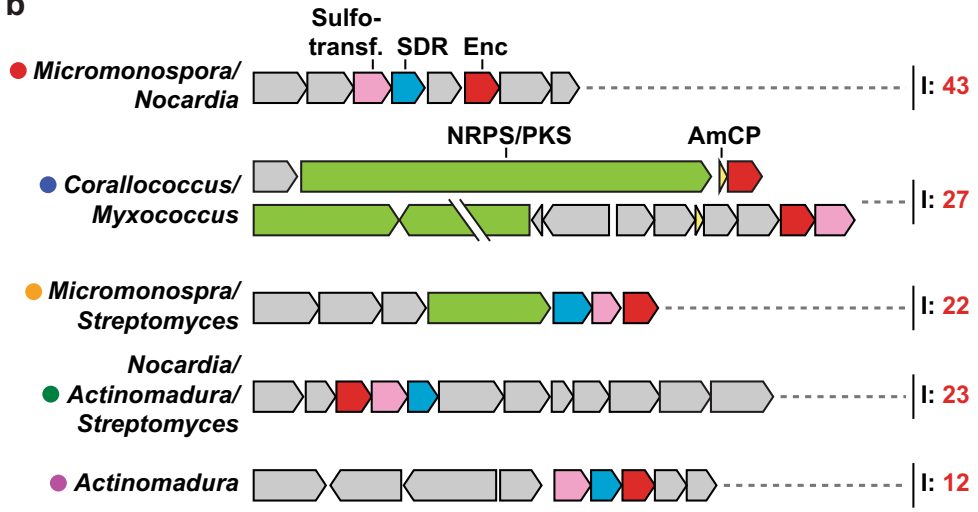

Mesorhizobium $\square \square \square \square \square \square \square \square-\ldots$

Fig. 5 Overview of Family 3 encapsulin systems. a SSN of Family 3 containing 138 nodes representing encapsulin capsid sequences clustered at $55 \%$ sequence identity. The inset shows chloromyxamide A, a natural product produced by a biosynthetic gene cluster encoding a Family 3 encapsulin found in Myxococcus sp. MCy $10608^{41}$. b Diversity of operon types encoding Family 3 encapsulins. Sulfotransferases, SDR-family oxidoreductases, non-ribosomal peptide synthetases (NRPSs)/polyketide synthases (PKSs), and amino-group carrier proteins (AmCPs) are commonly found in Family 3 operons. I number of identified operons.

MCy10608 (Fig. S8) ${ }^{41}$. This Myxococcus BGC was shown to produce a variety of chlorinated 6-chloromethyl-5methoxypipecolic acid-containing peptide natural products dubbed chloromyxamides. The chloromyxamide biosynthetic pathway and the role of the BGC-encoded Family 3 encapsulin are currently unknown. Based on gene annotations, putative biosynthetic pathways for all the other BGC classes have been proposed (Fig. S9, S10, S11 and S12). Given the presence of conserved pairs of sulfotransferases and SDRs in many of the identified BGCs, it is likely that the respective natural products will contain sulfated hydroxyl groups generated through the successive action of SDRs and sulfotransferases (Fig. S9) ${ }^{42}$. Some of the identified BGCs encode LysW-like AmCPs, suggesting that these biosynthetic pathways rely on covalently tethered intermediates, as observed in bacterial lysine and arginine biosynthesis (Fig. S10 $)^{43}$. Other BGCs contain large genes encoding NRPS or PKS multidomain enzymes responsible for non-ribosomal peptide and polyketide assembly (Fig. S11). The diversity of peptide bond-forming as well as peptide tailoring enzymes encoded in Family 3-associated BGCs suggests that they are capable of producing a structurally diverse set of peptide natural products.

A unique type of Family 3 encapsulin containing a C-terminal extension annotated as a major facilitator superfamily (MFS) domain--containing 4 to 5 predicted transmembrane helices--was identified in a number of Mesorhizobium spp. (Fig. S12 and Fig. S13). The respective BGCs further encode SDRs and enzymes commonly found in serine biosynthesis like phosphoserine aminotransferase, and phosphoserine phosphatase. These Mesorhizobium BGCs might be involved in the biosynthesis of a phosphorylated amino acid derivative (Fig. S12). While the role of the predicted transmembrane helices found in these Family 3 encapsulins is unknown, they may form a hydrophobic MFS-like gated channel surrounding the encapsulin pores (Fig. S13). Alternatively, they may mediate encapsulin-lipid membrane interactions or even recruit a lipid layer around the Family 3 encapsulin shell, similar to a viral envelope.

What role do Family 3 encapsulins play in the identified BGCs? Many of the tailoring enzymes found in Family 3 BGCs contain extended unannotated or possibly disordered regions at their $\mathrm{N}$ or C-termini. This may suggest that some of them are cargo proteins that are actively encapsulated, a theme observed for both
Family 1 and Family 2 systems. Active encapsulation of certain biosynthetic enzymes may allow Family 3 encapsulins to function as nanoscale reaction vessels and sequester reactive aldehyde or ketone intermediates (Fig. S8, S8, S10, S11 and S12) thus preventing potentially toxic side reactions in the cell cytoplasm. Similar molecular logic has been observed for bacterial microcompartments where a protein shell acts as a diffusion barrier for volatile or reactive pathway intermediates ${ }^{44}$.

Family 4 - A-domain encapsulins. A-domain Encapsulins are the most distinctive type of encapsulin-like system discovered in this study. They represent a highly truncated version of the HK97-fold and are predominantly found in genomes of hyperthermophilic Archaea. All so far sequenced Pyrococcus and Thermococcus genomes contain at least one but oftentimes two Adomain Encapsulin systems. Both Family 1 and Family 4 encapsulin-encoding genes were found in seven archaeal genomes, mostly Pyrococcus and Thermococcus, and account for 15\% of the identified A-domains. Outside Archaea, A-domain Encapsulins are only present in the two thermophilic Bacteroidetes genera Rubricoccus and Rhodothermus (Fig. S14). The fact that all organisms encoding Family 4 encapsulins are thermophilic anaerobes and were all isolated from submarine hydrothermal vents may implicate these systems in biological functions directly related to the extreme environmental conditions of these unique habitats.

SSN-analysis of all 95 identified Family 4 encapsulins revealed clear separation into 3 distinct clusters from now on referred to as Family 4 A, 4B and 4 C (Fig. 6a). Four conserved operon types could be identified based on the identity of the enzymatic components encoded upstream of the A-domain protein (Fig. 6b). Further, a subset of identified A-domain encapsulins, including all bacterial representatives, did not have any clearly associated enzymatic components and are thus referred to as Orphan/ Unknown. However, it should be noted that heme biosynthesis components are enriched in the genome neighborhood of bacterial A-domain Encapsulins (Fig. S14). The four conserved enzymatic components found in archaeal systems are: $[\mathrm{NiFe}]$ sulfhydrogenase (Hydrogenase, four subunits: $\alpha \beta \gamma \delta$ ), osmotically inducible protein $\mathrm{C}$ (OsmC), glyceraldehyde-3-phosphate dehydrogenase (GAPDH) and deoxyribose-phosphate aldolase (DeoC). Mapping these four operon types onto the SSN showed 
a

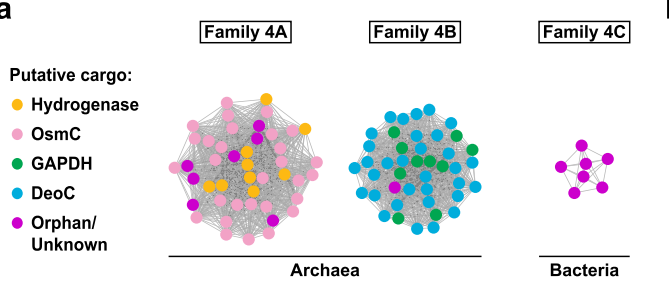

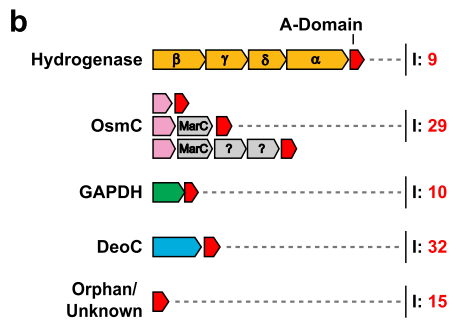

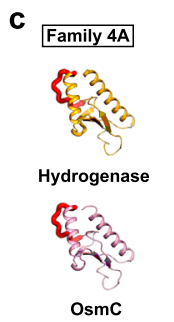

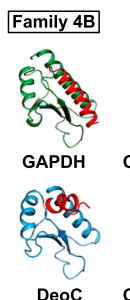

eoC

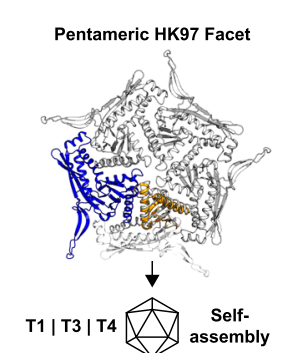

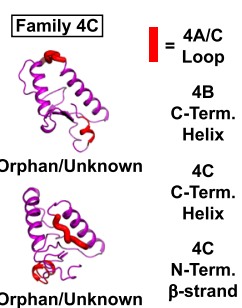

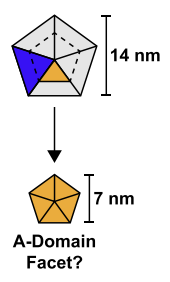

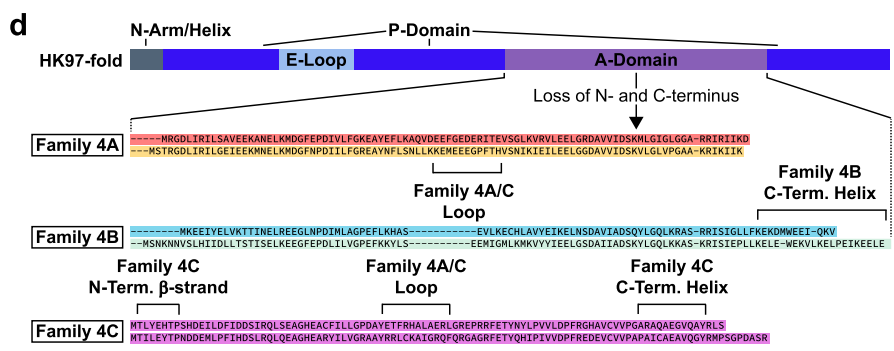

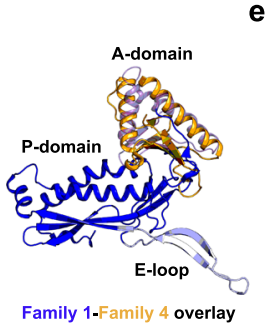

Fig. 6 Overview and analysis of Family 4 encapsulins. a SSN analysis of Family 4. Nodes represent 95 A-domain Encapsulins clustered at 38\% sequence identity. Nodes are colored by operon type based on associated enzyme components. b Overview of Family 4 operon types highlighting enzyme components (colored) and the number of identified systems (I). c Structural analysis of A-domain Encapsulin monomers based on homology modeling. Structural features distinguishing Family 4 A, 4B and 4C are highlighted in red. d Left: Sequence and structure of the HK97-fold and origin of A-domain Encapsulins. Loss of $\mathrm{N}$ - and $\mathrm{C}$-terminal domains results in a truncated protein corresponding to the A-domain of the HK97-fold. Distinguishing structural features of Family 4A, 4B and 4C shown in c are highlighted. Right: Structural comparison of HK97-fold (T1 Classical Encapsulin (3DKT), blue and purple) and A-domain Encapsulin (yellow). e Pentameric facet of a Family 1 encapsulin compared with an A-domain Encapsulin. A-domain Encapsulins may assemble into smaller pentameric facets about half the size of HK97-fold facets.

that Hydrogenase and OsmC operons are confined to Family $4 \mathrm{~A}$ while GAPDH and DeoC operons can only be found in Family 4B (Fig. 6a). Generally, each Pyrococcus and Thermococcus species encodes two separate A-domain Encapsulin systems, specifically, one Family 4A and one Family 4B operon.

A-domain Encapsulins are structurally similar to the A-domain of the HK97-fold. The crystal structure of a Family 4A Hydrogenase A-domain Encapsulin from Pyrococcus furiosus was solved, but not further characterized (PDB ID: 2PK8) ${ }^{45}$. The protein was $\mathrm{N}$-terminally His-tagged and crystallized as a dimer. Using $2 \mathrm{PK} 8$ as a threading template, the I-TASSER server ${ }^{46}$ was used to generate homology models of A-domain Encapsulins from Family 4A, 4B, and $4 \mathrm{C}$ as well as all operon types (Hydrogenase, OsmC, GAPDH and DeoC) (Fig. 6c). Similar to the A-domain of the HK97-fold ${ }^{23,24}$, A-domain Encapsulin monomers consist of two $\alpha$-helices surrounding a central fourstranded $\beta$-sheet called the $\beta$-hinge. The 3 subfamilies differ due to the presence of an $\mathrm{N}$-terminal $\alpha$-helix or an additional Cterminal $\beta$-strand as well as the presence or absence of an extended loop between the two main helices. Sequence similarity between A-domain Encapsulins and HK97-fold proteins is very low, however, based on structural alignments (Fig. 6d), it appears that large portions of the HK97-fold $\mathrm{N}$ - and C-terminal domains were lost, resulting in a contiguous stretch of about 100 amino acids representing the A-domain. All known HK97-fold proteins have the ability to self-assemble into pentameric C5 symmetrical complexes, also known as facets, that usually assemble further into icosahedral closed capsids (Fig. 6e) ${ }^{23,24}$. HK97-fold Adomains are also crucial for the formation of symmetrical pores at the five-fold symmetry axis in both Classical Encapsulins and viruses 23,24 . The two main helices of the A-domain form the major interaction interfaces between the five subunits of a facet. The conformational similarity of A-domain Encapsulins and HK97-fold proteins when part of a pentameric facet can be easily illustrated via structural alignments (Fig. 6e). We hypothesize that
A-domain Encapsulins should also be able to self-assemble into facets and potentially larger complexes. The fact that $2 \mathrm{PK} 8 \mathrm{did}$ crystallize as a dimer may be an artefact due to the presence of an N-terminal His-tag which could easily interfere with facet formation.

Family 4 Hydrogenase systems encode a four subunit [NiFe] hydrogenase as their enzymatic component. The specific [NiFe] hydrogenases associated with A-domain Encapsulins generally form cytoplasmic soluble heterotetrameric complexes ${ }^{47,48}$ and catalyze the reversible interconversion of $\mathrm{H}_{2}$ to two protons and two electrons ${ }^{49}$. The A-domain Encapsulin-associated [NiFe] hydrogenase of $P$. furiosus has been partially functionally characterized, however, this was done through whole cell measurements and heterologous expression experiments which did not yield any information about the associated A-domain Encapsulin ${ }^{50,51}$. In $P$. furiosus, this hydrogenase complex is known as sulfhydrogenase I (SHI) referring to its ability to act as a sulfur reductase, oxidizing $\mathrm{H}_{2}$ whilst simultaneously reducing elemental sulfur or polysulfides to hydrogen sulfide $\left(\mathrm{H}_{2} \mathrm{~S}\right)^{52,53}$. SHI has been proposed to primarily work in the direction of $\mathrm{H}_{2}$ formation in an NADPH-dependent manner ${ }^{54}$. It has been suggested that SHI mostly serves as a safety valve to remove excess reducing equivalents from the cytosol, thus playing an important role in maintaining intracellular redox homeostasis $52,55,56$.

The OsmC system encodes a single copy of the OsmC protein as its enzymatic component and often a MarC-like transmembrane protein, all located directly upstream of the A-domain Encapsulin. OsmC-type proteins are also known as organic hydroperoxide resistance (Ohr) proteins ${ }^{57}$. OsmC-like proteins are known to be organic hydroperoxidases and play important roles in microbial resistance against a broad range of fatty acid hydroperoxides and peroxynitrites generated as a result of oxidative and nitrosative stress ${ }^{58}$. OsmC proteins generally form dimeric structures containing a two-cysteine active site $e^{59-61}$. 
Peroxides are reduced to the corresponding alcohols and water with concomitant formation of a disulfide bond between the two active site cysteines ${ }^{62}$. After re-reduction, OsmC is ready for the next catalytic cycle. Studies indicate that the biological reductant of OsmC is dihydrolipoamide and not one of the more common cellular reducing agents like thioredoxin or glutathione ${ }^{63-65}$. It is unclear how MarC could be involved in the function of OsmC type A-domain Encapsulin systems ${ }^{66}$.

The GAPDH system consists of a gene encoding for a glyceraldehyde-3-phosphate dehydrogenase arranged in a twogene operon with the downstream A-domain component. GAPDH is a housekeeping gene present in all domains of life and is a key component of glycolysis and gluconeogenesis as well as other varied pathways and processes ${ }^{67-69}$. In Archaea of the genera Pyrococcus and Thermococcus, tetrameric GAPDH is part of the reversible modified Embden-Meyerhof-Parnas (EMP) pathway responsible for glycolysis and gluconeogenesis ${ }^{70-72}$. In the classical EMP pathway for sugar degradation in eukaryotes and bacteria, GAPDH catalyzes the reversible oxidation of glyceraldehyde-3-phosphate (GAP) to 1,3-bisphosphoglycerate (1,3BPG). In contrast, hyperthermophilic Archaea skip 1,3BPG formation and convert GAP directly to 3-phosphoglycerate (3PG) via enzymes only found in Archaea (GAPOR: GAP oxidoreductase or GAPN: non-phosphorylating GAP dehydrogenase) $)^{73-77}$. Pyrococcus and Thermococcus species encode GAPN which functions in the catabolic (glycolysis) direction while the single GAPDH encoded in their genomes, which is associated with an A-domain Encapsulin, is most highly expressed under gluconeogenic conditions and likely functions exclusively in the anabolic (gluconeogenesis) direction ${ }^{50,77-79}$. This likely implicates GAPDH A-domain Encapsulin operons in central carbon metabolism, specifically gluconeogenesis.

DeoC systems encode a deoxyribose-phosphate aldolase upstream of the A-domain component. DeoC forms a tetrameric complex and catalyzes the reversible reaction of 2-deoxy-D-ribose 5 -phosphate to GAP and acetaldehyde ${ }^{80,81}$. DeoC activity facilitates the utilization of exogenous nucleosides and nucleotides for energy generation where GAP and acetaldehyde can enter glycolysis and the citric acid cycle, respectively ${ }^{82,83}$. DeoC has also been shown to be upregulated under various stress conditions in Thermococcus species and other organisms which was hypothesized to indicate a redirection of carbon flux through DeoC and thus DNA precursor biosynthesis to maintain equilibrium between various catabolic and anabolic metabolic intermediates $^{84-86}$. Based on this analysis, we suggest that DeoC A-domain Encapsulin systems are involved in the utilization of nucleosides and nucleotides.

After discussing potential biological functions of A-domain Encapsulin systems, the function of the A-domain protein itself remains speculative. It is likely that A-domain Encapsulins fulfill a structural function in analogy to all other known HK97-fold proteins and that they retain the ability to self-assemble into higher order structures. We hypothesize that the respective enzymatic components of each operon type form complexes with the structural A-domain component. This is supported by a proteomics study carried out in $P$. furiosus that showed that GAPDH and the respective A-domain Encapsulin form a stable complex $^{48}$. Complex formation might be based on an interaction of the enzymatic component with one or multiple A-domain facets or even the encapsulation of the enzyme component inside a closed shell formed from self-assembled A-domain oligomers. What could be the specific functional role A-domain proteins play in these complexes? One possibility is that A-domain Encapsulins stabilize the respective enzymatic components through close association or encapsulation, in essence acting as specialized molecular chaperones ${ }^{87,88}$. This might result in increased thermal stability, increased resistance against oxidative stress and a prolonged productive lifetime of the associated enzyme complexes. A-domain Encapsulins might also protect enzymatic reaction intermediates from competing side reactions or sequester reactive or toxic intermediates inside a protein shell similar to what has been proposed for bacterial microcompartments ${ }^{44}$.

Pathogen-encoded encapsulins and their role in pathogenicity and virulence. Encapsulin systems can be found in a wide variety of prominent Gram-negative and Gram-positive pathogens. Family 1 and 2 encapsulins (peroxidase, Flp, and desulfurase systems) are found in pathogenic Escherichia coli, Klebsiella pneumoniae, and Acinetobacter baumannii, belonging to the highly virulent and antibiotic-resistant ESKAPE group of pathogens, responsible for the majority of life-threatening hospital-acquired infections worldwide ${ }^{89}$. Family 1 peroxidase operons are widely distributed in Mycobacteria, including $M$. tuberculosis and M. leprae, the causative agents of tuberculosis and leprosy, respectively ${ }^{90,91}$. Flp and desulfurase systems are both found in Burkholderia cepacia (pulmonary infections, cystic fibrosis) and Burkholderia pseudomallei (melioidosis) ${ }^{92}$ while Nocardia spp. (nocardiosis), Bordetella spp. (whooping cough), and Clostridium spp. (colitis, botulism, gangrene) encode peroxidase and Flp encapsulins ${ }^{93,94}$.

Most pathogen-encoded encapsulins are likely involved in stress resistance and nutrient utilization functions, often important for host invasion and proliferation in hostile environments like an infection site ${ }^{95-97}$. A direct link between $M$. tuberculosis oxidative stress resistance during infection and a Family 1 peroxidase system has recently been established which represents the first direct evidence of the involvement of encapsulins in pathogenicity and virulence ${ }^{11}$. In addition to stress resistance, it is possible that specialized encapsulin-based nutrient utilization systems - specifically for the two scarce and essential elements iron and sulfur - can increase pathogen fitness and proliferation, similar to the importance of bacterial microcompartment-based carbon and nitrogen source utilization systems for the pathogenicity of Salmonella typhimurium (food poisoning), Enterococcus faecalis (nosocomial infections), and Clostridium difficile (colitis) ${ }^{98-100}$. Iron storage encapsulin systems may further play a role in pathogenicity by influencing biofilm formation, which is linked to iron acquisition and homeostasis in ESKAPE pathogens ${ }^{101}$. Future efforts to characterize pathogen-associated encapsulin systems may yield novel targets for therapeutic intervention.

Phylogenetic analysis of encapsulins and related HK97-fold proteins. All four families of encapsulins discussed above belong to the Pfam clan CL0373. A Pfam clan is a collection of related Pfam families ${ }^{19}$. Membership within a Pfam clan is determined by up to four independent pieces of evidence: related structure/ fold, related function, significant matching of sequences to HMMs from separate Pfam families, and pairwise profile-profile HMM alignments based on HHsearch ${ }^{102}$. The fact that Pfam clans are meant to contain only Pfam families that share a common evolutionary origin ${ }^{19}$ is a first indication that all four encapsulin families are in fact evolutionarily related to the other HK97-fold proteins, all representing phage and virus capsid proteins, contained within CL0373. To further investigate the relationship between encapsulin-like proteins and virus capsids, we carried out a detailed phylogenetic analysis of CL0373. Due to the generally low sequence similarity among virus capsid proteins and between encapsulin families which makes multiple sequence alignments difficult, we based our analysis on the most conserved 


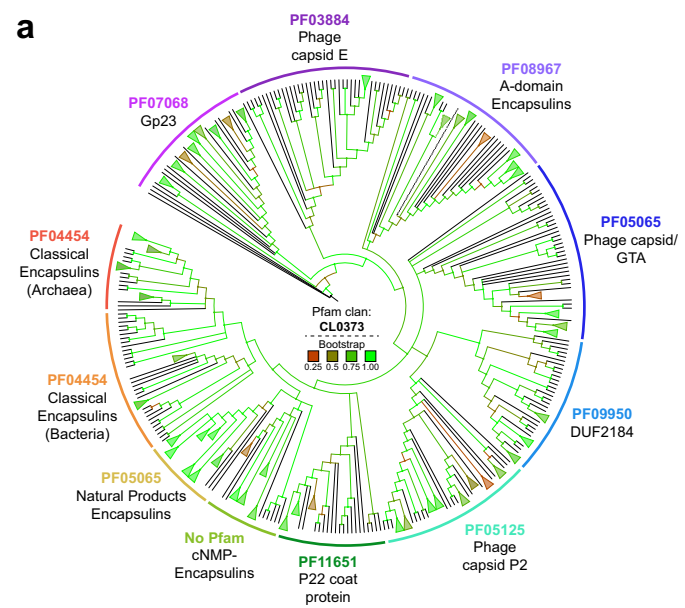

b

c

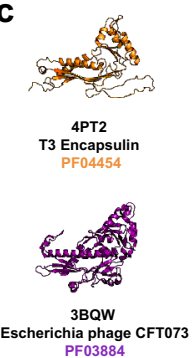

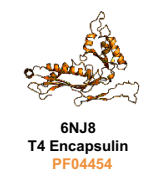

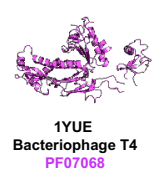

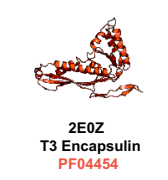

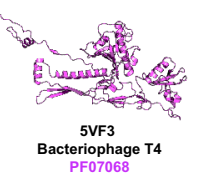

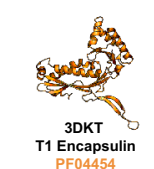

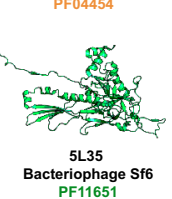

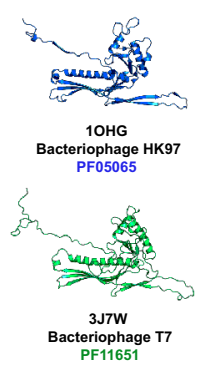

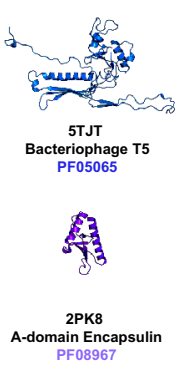

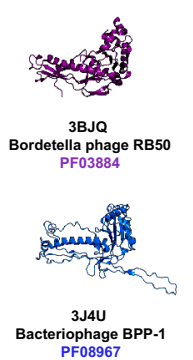

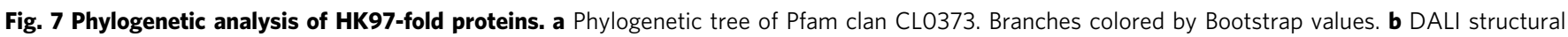
comparisons of representative CL0373 structures of each family with available structures. Left: dendrogram based on pairwise Z score comparisons. Right: matrix/heatmap representation of $Z$ scores based on pairwise comparisons. The color scale indicates $Z$ scores. Pfam families colored as in (a). c

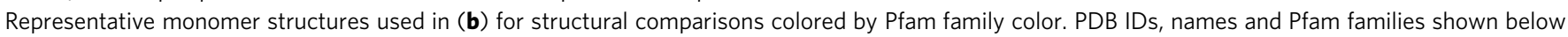
each monomer structure.

regions of the HK97-fold, specifically the A-domain and neighboring regions belonging to parts of the HK97-fold P-domain (Fig. 6d). The resulting phylogenetic tree showed relatively confident bootstrap values and allowed us to investigate the relationships between all members of CL0373 in more detail (Fig. 7a). All encapsulin families except Family 4 (A-domain Encapsulins) are more closely related to one another than to other HK97-type proteins indicating that they might all share a recent common ancestor. The P22 coat protein family (PF11651) seems to be the virus capsid protein family most closely related to Family 1, 2 and 3 encapsulins. Classical Encapsulins (Family 1) found in both Bacteria and Archaea are more closely related to one another than to any other HK97-fold proteins. This suggests inter-domain horizontal transfer of Family 1 encapsulin systems, likely from Bacteria to Archaea, which is a well-documented phenomenon ${ }^{103,104}$. A-domain Encapsulins (Family 4) appear to be more evolutionarily distinct from the other encapsulin families and to be more closely related to other HK97-fold capsid proteins. The Gp23 family (PF07068) generally found in T4-like bacteriophages seems to be the most distantly related Pfam family compared with Family 1, 2 and 3 encapsulins. Our sequencebased analysis suggests that encapsulins share common ancestry with all HK97-fold families contained within CL0373 and indicates that encapsulin systems likely evolved from viruses, specifically from members of the widespread virus order Caudovirales $^{105}$.

Further analysis of CL0373 members was carried out via structural comparisons of representatives of all investigated Pfam families for which structures were available (Fig. 7b). Pairwise structural similarities were evaluated using the DALI Z score ${ }^{106}$. The $\mathrm{Z}$ score is a measure of the overall quality of a given structural comparison. All-against-all structural comparisons showed that Family 1 encapsulins form an apparent monophyletic cluster while the single available Family 2 encapsulin $(6 \mathrm{X} 8 \mathrm{~T})^{8}$ was more similar to PF05065. It should be noted that 6X8T represents a Family 2 encapsulin without a cNMP-binding domain. No structure of the more ubiquitous cNMP-containing Family 2 systems is currently available. The other viral HK97-type proteins are more divergent compared to the available encapsulin structures (Fig. 7b). Further visual inspection of sample structures (Fig. 7c) reveals that Family 1 encapsulins do not possess an extended N-arm which is present in the majority of other HK97fold proteins. Instead, they possess an N-terminal helix which forms part of the binding pocket for the targeting peptide of cargo proteins ${ }^{10,15}$. Some of the sample structures additionally possess insertion domains that are present in the E-loop (PF07068) 23,24. Family 1 encapsulins generally appear more compact with a shorter central P-domain helix and shorter E-loop. In accordance with the DALI structural comparison, the Family 2 encapsulin example appears structurally more similar to the phage capsid family PF05065 than to Family 1 encapsulins. As discussed above, Family 4 encapsulins are structurally similar to the A-domain of the HK97-fold ${ }^{45}$. No Family 3 structures have been solved at the time of writing. However, some Family 3 members have been annotated as belonging to Pfam family PF05065 which may indicate that they are more structurally similar to Family 2 than Family 1 encapsulins.

Both our sequence- and structure-based analysis argues for a viral origin of encapsulin systems likely via domestication of prophage HK97-type capsid proteins. This is also in agreement with the fact that HK97-fold viruses are ubiquitous and found as proviruses and prophages in the genomes of members of all 
domains of life while encapsulins show a narrower distribution ${ }^{9,105}$. Considering that one of the current hypotheses regarding the origin of viral capsid proteins is a scenario where they ultimately derive from cellular protein folds of ancient or extinct cellular lineages ${ }^{107}$, the HK97-fold might have undergone a re-recruitment, and as part of encapsulin systems has now returned to its cellular origin.

\section{Conclusion}

The curated set of encapsulin-like systems discovered and analyzed here, sheds light on the true functional diversity of microbial protein compartments. Proposed encapsulin functions include roles as reaction spaces for various anabolic (Family 2 and 3 ) and catabolic (Family 2) processes, storage compartments (Family 1), enzyme regulatory systems (Family 2 and 4 ) as well as chaperones (Family 4). Encapsulins are found in aerobic and anaerobic microbes that occupy nearly all terrestrial and aquatic habitats as well as host-associated niches. Additionally, encapsulins are widespread in bacterial and archaeal extremophiles, specifically (hyper)thermophiles and acidophiles. While we have discovered a comprehensive and curated set of encapsulin systems based on the available data, the ever-increasing throughput of genomic and metagenomic sequencing as well as rapid advances in computational tool development likely ensure that more encapsulin systems will be discovered in future studies. The evolutionary scenario outline above, where encapsulin systems are the result of the molecular domestication of phage capsid proteins by cellular hosts, is further supported by the existence of transitional systems like the Family 1 encapsulin found in Sulfolobus solfataricus whose genetic context indicates that it used to be part of a now defective prophage ${ }^{36}$. It is possible that other viral capsid protein folds may also have undergone a similar recruitment process and now serve specific host metabolic functions. This idea is supported by the recent description of the involvement of the retrovirus-like capsid protein Arc in interneuron nucleic acid transport ${ }^{108}$. In conclusion, our study establishes encapsulins as a ubiquitous and diverse class of protein compartmentalization systems and lays the groundwork for future experimental studies aimed at better understanding the physiological roles and biomedical relevance of encapsulins.

\section{Methods}

Genome-mining searches for encapsulin-like systems. Family 1 and Family 4 encapsulins were identified using the Enzyme Function Initiative-Enzyme Similarity Tool (EFI-EST) Families search function against the full UniProt database to filter for sequences corresponding to Pfam families PF04454 (Family 1) or PF08957 (Family 4) in May 202020,109-112. Family $4 \mathrm{C}$ encapsulins were identified through additional blastp searches against the NCBI_nr database using the initially identified Family $4 \mathrm{~A}$ and $4 \mathrm{~B}$ hits as queries. Family 2 encapsulins were initially identified based on using the EFI-EST Sequence BLAST function with a previously identified encapsulin as a query (WP_011055154.1) ${ }^{113}$. Searches were carried out against the UniProt database with an E-value of 5. This allowed us to generate an initial SSN of 1,770 sequences. To expand the dataset, we aligned 40 edge sequences from the initial dataset containing both Family $2 \mathrm{~A}$ and 2B encapsulins using Clustal Omega v1.2.2 in the Geneious Prime software package with fast clustering (mBed algorithm) and a cluster size of 100 for mBed guide trees. Sequences were truncated to only contain the C-terminal capsid component - removing the putative cNMP-binding domain--and used to generate an initial HMM model using the hmmbuild function of the HMMER3 software package ${ }^{114,115}$. This HMM was then used as an input for the HMMER search tool in the MPI Bioinformatics Toolkit (https://toolkit.tuebingen.mpg.de/). Searches were carried out against the UniProt_Trembl database in May 2020 using an E-value cutoff of 10, 0 MSA enrichment iterations in HHblits, and a maximum of 10,000 target hits ${ }^{114,116,117}$. Family 3 encapsulins were identified by searching a previously identified putative encapsulin from Myxococcus (UniProt ID: A0A346D7L6) ${ }^{41}$ against the UniProt database using the EFI-EST Sequence BLAST function with an E- value threshold of 1 in February 2021. The resulting datasets generated from these initial searches contained the following numbers of sequences: Family 1: 2,540, Family 2: 3,859, Family 3: 215, Family 4: 95. Sequences labeled as fragments and unclassified sequences with superkingdoms labeled as metagenome were excluded. Family 1, 2, and 3 datasets were significantly contaminated with bacteriophage capsid proteins.
To remove phage contamination in the Family 1 and 2 datasets, custom Blast databases were generated containing proteins encoded within $10 \mathrm{~kb}$ upstream and downstream of each identified capsid gene. The custom Blast databases were then searched against proteomes of HK97-type phages and a broad dataset of prokaryotic dsDNA viruses (proteome IDs: UP000002576 and UP000391682) using blastp with default settings with an E-value threshold of 0.1 . Proteins identified as phage-related were excluded from the datasets. Because the Family 3 encapsulin dataset was much smaller than Family 1 and Family 2, phage proteins could be easily filtered manually by removing genome neighborhoods containing phageassociated Pfam domains (PF0860, PF03354, PF04586, PF00589, PF05135). All datasets were then further manually curated to exclude any remaining genome neighborhoods containing phage-related proteins. The final curated datasets contained the following number of sequences for each family: Family 1: 2,383, Family 2: 3,523, Family 3: 132, Family 4: 95 (Supplementary Data 1).

\section{Phylogenetic analyses and construction of phylogenetic trees}

Encapsulin distribution in prokaryotic phyla. An initial diagram of the phylogenetic distribution of prokaryotes was constructed from a previously published maximum likelihood tree of ribosomal protein alignments using the iTOL server ${ }^{118,119}$. Branches corresponding to Eukaryotes were removed, display mode set to circular, and clades were collapsed to a threshold of $<0.65 \mathrm{BRL}$. Branches were then annotated manually to highlight encapsulin containing phyla.

Encapsulins and related HK97-fold proteins. To infer phylogenetic relationships between encapsulin-like proteins and other HK97-type proteins, the Phage-coat Pfam clan CL0373 was used as a starting point ${ }^{19}$. Sequences from all families found within CL0373 that contained more than 10 members were used. The following Pfam families were considered with the number of sequences used shown in parentheses: DUF1884/Family 4 encapsulins PF08967 (40), DUF2184 PF09950 (37), Gp23 PF07068 (40), Linocin_M18/Family 1 encapsulins PF04454 (68), P22_CoatProtein PF11651 (40), Phage_cap_E PF03864 (40), Phage_cap_P2 PF05125 (40) and Phage_capsid PF05065 (40). Sequences were selected from the Seed and Full alignments of each Pfam family. Sequences from the following protein families that had no Pfam designation were additionally included in the analysis: Family 2 encapsulins (40) and Family 3 encapsulins (40). Sequences of putative Gene Transfer Agents belonging to family PF05065 were also included (29) ${ }^{120}$. Alignments, sequence curation and phylogenetic inference analyses were carried out using the NGPhylogeny.fr server ${ }^{121}$. A custom workflow using the following tools and parameters was used. For multiple sequence alignment, MAFFT ${ }^{122}$ was utilized with standard parameters; for alignment curation, $\mathrm{BMGE}^{123}$ was used with a maximum entropy threshold of 0.75 and otherwise standard parameters; for tree inference, PhyML+SMS ${ }^{124}$ was used with standard parameters; for tree visualization, iTOL ${ }^{119}$ was used with the following parameters deviating from the pre-set: display mode: circular, branch lengths: ignore, bootstraps: display as color with range 0 to 1 , auto collapse clades: $\mathrm{BRL}<0.5$. The sequence most distant to Family 1 encapsulins was used as the outgroup: J7HY26 (PF07068).

Terpene cyclases and polyprenyl transferases. To analyze the evolutionary relationships and diversity of terpenoid-related enzymes identified in Family 2 encapsulin operons, separate multiple sequence alignments and phylogenetic inference analyses were carried out for 530 terpene cyclase (all newly identified) and 122 polyprenyl transferase (97 newly identified, 25 already experimentally characterized) ${ }^{125}$ sequences (Supplementary Data 1). Already characterized polyprenyl transferase sequences were incorporated into our analysis to infer the putative substrate range of newly identified sequences. A custom workflow on the NGPhylogeny.fr server for sequence alignments, curation, and phylogenetic inference was used. MAFFT was utilized for multiple sequence alignments with standard parameters; alignment curation was done via BMGE and standard parameters; for tree inference, PhyML+SMS was employed using standard parameters; for phylogenetic tree visualization, iTOL was used with the following non standard parameters: display mode: unrooted, branch lengths: ignore, bootstraps: display as color with range 0 to 1 .

Sequence similarity network analysis. Sequence similarity networks (SSNs) were calculated using the EFI-ESI server $20,109,126$. Initial SSNs were generated for each family with edge E-values of 5 and alignment thresholds corresponding to approximately $40 \%$ sequence identity. SSNs were visualized in Cytoscape v3.8 $8^{127}$ using the yFiles organic layout and were then filtered to the following percent identity thresholds to optimize cluster separation and visual presentation: Family 1: 49\%, Family 2: 70\%, Family 3: 55\%, Family 4: 38\% (Supplementary Data 2). Nodes were colored according to cargo type for Family 1,2, and 4 encapsulins. Family 3 encapsulin nodes were colored according to natural product gene cluster type.

Genome neighborhood analysis. Genome neighborhood analysis was performed using the EFI-GNT server with EFI-ESI-generated and Cytoscape-curated network files (xgmml format) as inputs resulting in computed genome neighborhoods extending 20 open reading frames up- and downstream of the identified encapsulin-encoding genes ${ }^{20,109,126}$. 
To identify Family 1 cargo proteins, we first generated a custom database of all proteins encoded within 5000 bp up- and downstream of identified Family 1 encapsulin genes and used blastp to search for the Family 1 targeting peptide consensus sequence (SDGSLGIGSLKRS) ${ }^{9}$. Blastp parameters were automatically adjusted for short input sequences and an E-value of 200,000 was used. HMM templates representative of each cargo class identified through initial blastp searches were then generated and used as inputs for HMMsearch. The resulting set of cargo hits was then classified based on their Pfam or Interpro annotation. If no Pfam or Interpro annotation was present, cargo proteins were annotated based on sequence similarity. Identified cargo proteins that did not have corresponding NCBI or UniProt accession codes were labeled as putative. Manually curated cargo proteins that were not identified by any of the above search methods but located immediately adjacent to an encapsulin gene in the GNN or in the NCBI Nucleotide graphic interface were labeled as manually curated.

Family 2 cargo proteins were identified by constructing a custom database of all proteins encoded within 20 open reading frames of the identified Family 2 encapsulins, then HMMs were constructed from representative terpene cyclases, polyprenyl transferases, cysteine desulfurases, and xylulose kinases using HHbuild ${ }^{114}$. The resulting HMMs were then used to query our custom Family 2 database via HMMsearch. Identified cargo proteins of encapsulins not present in the ENA database were curated manually.

Family 3 and Family 4 encapsulins were manually inspected for putative cargo proteins and operon similarity using EFI-GNT-generated genome neighborhood diagrams.

\section{Protein homology models and protein structure analysis}

General. General protein structure editing and visualization were done using UCSF Chimera $^{128}$, UCSF ChimeraX ${ }^{129}$ and PyMOL.

Homology models. All protein homology models used for classification and analysis of Family 4 encapsulins were generated using the I-TASSER Protein Structure \& Function Prediction server ${ }^{46}$ with standard parameters. The following sequences were used as inputs: Family 4A: F0LMI5; Family 4B: F0LIR3 and O59495; Family 4C:

$\mathrm{A} 0 \mathrm{~A} 1 \mathrm{M} 6 \mathrm{P} 7 \mathrm{GO}$ and $\mathrm{A} 0 \mathrm{~A} 2 \mathrm{H} 0 \mathrm{JLL} 0$. In all cases, $2 \mathrm{PK} 8$ was found to be the best template.

Structure comparisons and similarity analysis. Structure comparisons between different HK97-type proteins were carried out on the DALI server ${ }^{106}$ using the following representative experimentally determined structures: PF04454: 4PT2, 6NJ8, 2E0Z and 3DKT; no Pfam (T1 Family 2A encapsulin): 6X8T; PF03884: 3BJQ and 3BQW; PF05065: 1OHG and 5TJT; PF07068: 1YUE and 5VF3; PF11651: 5L35 and 3J7W; PF08967: 2PK8; PF08967: 3J40 and 3J4U. Structural similarities between the selected proteins were evaluated based on the DALI Z score, which represents a measure of the quality of the overall structural alignment. For structure alignment visualization, structural similarity matrices resulting from all-against-all structure comparisons and the respective dendrograms were generated using the all-againstall structure comparison tool on the DALI server.

Analysis of disordered protein sequences. Sequence disorder analyses were carried out using the Disopred 3 server ${ }^{130}$ for the following representative proteins for each Family 2B cargo class: CD: A0A010WJT9, PT: A0A0B5EUR5, TC: 2MIBS-like: Q9F1Y6, TC-GS-like: A0A3D0QW52. Disopred3 outputs were visualized using GraphPad Prism v9.0.2.

Reporting Summary. Further information on research design is available in the Nature Research Reporting Summary linked to this article.

\section{Data availability}

Databases used in this study: European Nucleotide Archive (ENA, https://www.ebi.ac.uk/ ena/browser/home), NCBI Nucleotide database (https://www.ncbi.nlm.nih.gov/nuccore), NCBI Protein database (https://www.ncbi.nlm.nih.gov/protein), NCBI Genome database (https://www.ncbi.nlm.nih.gov/genome), UniProt database (https://www.uniprot.org/). An annotated and curated spreadsheet of all identified encapsulins and cargo proteins is available as Supplementary Data 1. Annotated SSNs for each encapsulin family are available as Supplementary Data 2.

Received: 10 June 2021; Accepted: 12 July 2021;

Published online: 06 August 2021

\section{References}

1. Diekmann, Y. \& Pereira-Leal, J. B. Evolution of intracellular compartmentalization. Biochem J. 449, 319-331 (2013).

2. Gabaldon, T. \& Pittis, A. A. Origin and evolution of metabolic sub-cellular compartmentalization in eukaryotes. Biochimie 119, 262-268 (2015).
3. Cornejo, E., Abreu, N. \& Komeili, A. Compartmentalization and organelle formation in bacteria. Curr. Opin. Cell Biol. 26, 132-138 (2014).

4. Greening, C. \& Lithgow, T. Formation and function of bacterial organelles. Nat Rev Microbiol, https://doi.org/10.1038/s41579-020-0413-0 (2020).

5. Nichols, R. J., Cassidy-Amstutz, C., Chaijarasphong, T. \& Savage, D. F. Encapsulins: molecular biology of the shell. Crit. Rev. Biochem Mol. Biol. 52, 583-594 (2017).

6. Giessen, T. W. Encapsulins: microbial nanocompartments with applications in biomedicine, nanobiotechnology and materials science. Curr. Opin. Chem. Biol. 34, 1-10 (2016).

7. Jones, J. A. \& Giessen, T. W. Advances in encapsulin nanocompartment biology and engineering. Biotechnol. Bioeng. 118, 491-505 (2021).

8. Nichols, R. J. et al. Discovery and characterization of a novel family of prokaryotic nanocompartments involved in sulfur metabolism. Elife 10, https://doi.org/10.7554/eLife.59288 (2021).

9. Giessen, T. W. \& Silver, P. A. Widespread distribution of encapsulin nanocompartments reveals functional diversity. Nat. Microbiol 2, 17029 (2017).

10. Sutter, M. et al. Structural basis of enzyme encapsulation into a bacterial nanocompartment. Nat. Struct. Mol. Biol. 15, 939-947 (2008).

11. Lien, K. A. et al. A nanocompartment containing the peroxidase DypB contributes to defense against oxidative stress in $<\mathrm{em}>\mathrm{M}$. tuberculosis $</ \mathrm{em}>$. bioRxiv, 2020.2008.2031.276014, https://doi.org/10.1101/2020.08.31.276014 (2020).

12. McHugh, C. A. et al. A virus capsid-like nanocompartment that stores iron and protects bacteria from oxidative stress. EMBO J. 33, 1896-1911 (2014).

13. Contreras, H. et al. Characterization of a Mycobacterium tuberculosis nanocompartment and its potential cargo proteins. J. Biol. Chem. 289, 18279-18289 (2014).

14. He, D. et al. Conservation of the structural and functional architecture of encapsulated ferritins in bacteria and archaea. Biochem J. 476, 975-989 (2019).

15. Giessen, T. W. et al. Large protein organelles form a new iron sequestration system with high storage capacity. Elife 8, https://doi.org/10.7554/eLife.46070 (2019).

16. Tracey, J. C. et al. The discovery of twenty-eight new encapsulin sequences, including three in Anammox bacteria. Sci. Rep. 9, 20122 (2019).

17. Altenburg, W. J., Rollins, N., Silver, P. A. \& Giessen, T. W. Exploring targeting peptide-shell interactions in encapsulin nanocompartments. Sci. Rep. 11, 4951 (2021).

18. UniProt, C. UniProt: a worldwide hub of protein knowledge. Nucleic Acids Res. 47, D506-D515 (2019).

19. Finn, R. D. et al. Pfam: clans, web tools and services. Nucleic Acids Res. 34, D247-251 (2006).

20. Zallot, R., Oberg, N. \& Gerlt, J. A. The EFI web resource for genomic enzymology tools: leveraging protein, genome, and metagenome databases to discover novel enzymes and metabolic pathways. Biochemistry 58, 4169-4182 (2019).

21. Ward, A. C. \& Allenby, N. E. Genome mining for the search and discovery of bioactive compounds: the Streptomyces paradigm. FEMS Microbiol Lett 365, https://doi.org/10.1093/femsle/fny240 (2018).

22. Bader, C. D., Panter, F. \& Muller, R. In depth natural product discovery Myxobacterial strains that provided multiple secondary metabolites. Biotechnol. Adv. 39, 107480 (2020).

23. Suhanovsky, M. M. \& Teschke, C. M. Nature's favorite building block: deciphering folding and capsid assembly of proteins with the HK97-fold. Virology 479-480, 487-497 (2015).

24. Duda, R. L. \& Teschke, C. M. The amazing HK97 fold: versatile results of modest differences. Curr. Opin. Virol. 36, 9-16 (2019)

25. Kim, S. J. \& Shoda, M. Purification and characterization of a novel peroxidase from Geotrichum candidum dec 1 involved in decolorization of dyes. Appl Environ. Microbiol 65, 1029-1035 (1999).

26. Ahmad, M. et al. Identification of DypB from Rhodococcus jostii RHA1 as a lignin peroxidase. Biochemistry 50, 5096-5107 (2011).

27. Rahmanpour, R. \& Bugg, T. D. Assembly in vitro of Rhodococcus jostii RHA1 encapsulin and peroxidase DypB to form a nanocompartment. FEBS J. 280, 2097-2104 (2013)

28. Tang, Y. et al. Cryo-EM structure of Mycobacterium smegmatis DyP-loaded encapsulin. Proc. Natl. Acad. Sci. USA 118, https://doi.org/10.1073/ pnas.2025658118 (2021).

29. He, D. et al. Structural characterization of encapsulated ferritin provides insight into iron storage in bacterial nanocompartments. Elife 5, https://doi. org/10.7554/eLife.18972 (2016).

30. LaFrance, B. et al. The encapsulin from Thermatoga maritima is a flavoprotein with a symmetry matched ferritin-like cargo protein. BioRxiv, 2021.2004.2026.441214, https://doi.org/10.1101/2021.04.26.441214 (2021).

31. Yao, H. et al. The structure of the BfrB-Bfd complex reveals protein-protein interactions enabling iron release from bacterioferritin. J. Am. Chem. Soc. 134, 13470-13481 (2012). 
32. Okamoto, $\mathrm{Y}$. et al. H2O2-dependent substrate oxidation by an engineered diiron site in a bacterial hemerythrin. Chem. Commun. (Camb.) 50, 3421-3423 (2014).

33. Alvarez-Carreno, C., Alva, V., Becerra, A. \& Lazcano, A. Structure, function and evolution of the hemerythrin-like domain superfamily. Protein Sci. 27, 848-860 (2018).

34. Rivera, M. Bacterioferritin Structure, dynamics, and protein-protein interactions at play in iron storage and mobilization. Acc. Chem. Res. 50, 331-340 (2017).

35. Akita, F. et al. The crystal structure of a virus-like particle from the hyperthermophilic archaeon Pyrococcus furiosus provides insight into the evolution of viruses. J. Mol. Biol. 368, 1469-1483 (2007).

36. Heinemann, J. et al. Fossil record of an archaeal HK97-like provirus. Virology 417, 362-368 (2011).

37. Medema, M. H. et al. A predicted physicochemically distinct sub-proteome associated with the intracellular organelle of the anammox bacterium Kuenenia stuttgartiensis. BMC Genomics 11, 299 (2010).

38. Ferousi, C. et al. Identification of the type II cytochrome $\mathrm{c}$ maturation pathway in anammox bacteria by comparative genomics. BMC Microbiol 13, 265 (2013)

39. Hidese, R., Mihara, H. \& Esaki, N. Bacterial cysteine desulfurases: versatile key players in biosynthetic pathways of sulfur-containing biofactors. Appl Microbiol Biotechnol. 91, 47-61 (2011).

40. Kessler, D. Enzymatic activation of sulfur for incorporation into biomolecules in prokaryotes. FEMS Microbiol Rev. 30, 825-840 (2006).

41. Gorges, J. et al. Structure, total synthesis, and biosynthesis of chloromyxamides: myxobacterial tetrapeptides featuring an uncommon 6Chloromethyl-5-methoxypipecolic acid building block. Angew. Chem. Int Ed. Engl. 57, 14270-14275 (2018).

42. Kavanagh, K. L., Jornvall, H., Persson, B. \& Oppermann, U. Medium- and short-chain dehydrogenase/reductase gene and protein families: the SDR superfamily: functional and structural diversity within a family of metabolic and regulatory enzymes. Cell Mol. Life Sci. 65, 3895-3906 (2008).

43. Ouchi, T. et al. Lysine and arginine biosyntheses mediated by a common carrier protein in Sulfolobus. Nat. Chem. Biol. 9, 277-283 (2013).

44. Kerfeld, C. A., Aussignargues, C., Zarzycki, J., Cai, F. \& Sutter, M. Bacterial microcompartments. Nat. Rev. Microbiol 16, 277-290 (2018).

45. Kelley, L. L. et al. Structure of the hypothetical protein PF0899 from Pyrococcus furiosus at 1.85 A resolution. Acta Crystallogr Sect. F. Struct. Biol. Cryst. Commun. 63, 549-552 (2007).

46. Zheng, W., Zhang, C., Bell, E. W. \& Zhang, Y. I-TASSER gateway: a protein structure and function prediction server powered by XSEDE. Future Gener. Comput Syst. 99, 73-85 (2019).

47. Chandrayan, S. K. et al. Engineering hyperthermophilic archaeon Pyrococcus furiosus to overproduce its cytoplasmic [NiFe]-hydrogenase. J. Biol. Chem. 287, 3257-3264 (2012).

48. Menon, A. L. et al. Novel multiprotein complexes identified in the hyperthermophilic archaeon Pyrococcus furiosus by non-denaturing fractionation of the native proteome. Mol. Cell Proteom. 8, 735-751 (2009).

49. Ash, P. A., Kendall-Price, S. E. T. \& Vincent, K. A. Unifying activity, structure, and spectroscopy of [NiFe] hydrogenases: combining techniques to clarify mechanistic understanding. Acc. Chem. Res. 52, 3120-3131 (2019).

50. Schut, G. J., Brehm, S. D., Datta, S. \& Adams, M. W. Whole-genome DNA microarray analysis of a hyperthermophile and an archaeon: Pyrococcus furiosus grown on carbohydrates or peptides. J. Bacteriol. 185, 3935-3947 (2003).

51. Sun, J., Hopkins, R. C., Jenney, F. E., McTernan, P. M. \& Adams, M. W. Heterologous expression and maturation of an NADP-dependent [NiFe]hydrogenase: a key enzyme in biofuel production. PLoS ONE 5, e10526 (2010).

52. Chou, C. J. et al. Impact of substrate glycoside linkage and elemental sulfur on bioenergetics of and hydrogen production by the hyperthermophilic archaeon Pyrococcus furiosus. Appl Environ. Microbiol 73, 6842-6853 (2007).

53. Fiala, G. \& Stetter, K. O. Pyrococcus furiosus sp. nov. represents a novel genus of marine heterotrophic archaebacteria growing optimally at $100^{\circ} \mathrm{C}$. Arch. Microbiol 145, 56-61 (1986).

54. Bryant, F. O. \& Adams, M. W. Characterization of hydrogenase from the hyperthermophilic archaebacterium, Pyrococcus furiosus. J. Biol. Chem. 264, 5070-5079 (1989).

55. Silva, P. J. et al. Enzymes of hydrogen metabolism in Pyrococcus furiosus. Eur. J. Biochem 267, 6541-6551 (2000).

56. van Haaster, D. J., Silva, P. J., Hagedoorn, P. L., Jongejan, J. A. \& Hagen, W. R. Reinvestigation of the steady-state kinetics and physiological function of the soluble NiFe-hydrogenase I of Pyrococcus furiosus. J. Bacteriol. 190, 1584-1587 (2008).

57. Mongkolsuk, S., Praituan, W., Loprasert, S., Fuangthong, M. \& Chamnongpol, S. Identification and characterization of a new organic hydroperoxide resistance (ohr) gene with a novel pattern of oxidative stress regulation from Xanthomonas campestris pv. phaseoli. J. Bacteriol. 180, 2636-2643 (1998).
58. Alegria, T. G. et al. Ohr plays a central role in bacterial responses against fatty acid hydroperoxides and peroxynitrite. Proc. Natl Acad. Sci. USA 114, E132-E141 (2017).

59. Rehse, P. H., Ohshima, N., Nodake, Y. \& Tahirov, T. H. Crystallographic structure and biochemical analysis of the Thermus thermophilus osmotically inducible protein C. J. Mol. Biol. 338, 959-968 (2004).

60. Choi, I. G. et al. Crystal structure of a stress inducible protein from Mycoplasma pneumoniae at 2.85 A resolution. J. Struct. Funct. Genomics 4 , 31-34 (2003)

61. Lesniak, J., Barton, W. A. \& Nikolov, D. B. Structural and functional characterization of the Pseudomonas hydroperoxide resistance protein Ohr. EMBO J. 21, 6649-6659 (2002)

62. Oliveira, M. A. et al. Structural insights into enzyme-substrate interaction and characterization of enzymatic intermediates of organic hydroperoxide resistance protein from Xylella fastidiosa. J. Mol. Biol. 359, 433-445 (2006).

63. Cussiol, J. R., Alegria, T. G., Szweda, L. I. \& Netto, L. E. Ohr (organic hydroperoxide resistance protein) possesses a previously undescribed activity, lipoyl-dependent peroxidase. J. Biol. Chem. 285, 21943-21950 (2010).

64. Meunier-Jamin, C., Kapp, U., Leonard, G. A. \& McSweeney, S. The structure of the organic hydroperoxide resistance protein from Deinococcus radiodurans. Do conformational changes facilitate recycling of the redox disulfide? J. Biol. Chem. 279, 25830-25837 (2004).

65. Cussiol, J. R., Alves, S. V., de Oliveira, M. A. \& Netto, L. E. Organic hydroperoxide resistance gene encodes a thiol-dependent peroxidase. J. Biol. Chem. 278, 11570-11578 (2003).

66. McDermott, P. F. et al. The marC gene of Escherichia coli is not involved in multiple antibiotic resistance. Antimicrob. Agents Chemother. 52, 382-383 (2008).

67. Seidler, N. W. Basic biology of GAPDH. Adv. Exp. Med. Biol. 985, 1-36 (2013).

68. Seidler, N. W. GAPDH and intermediary metabolism. Adv. Exp. Med. Biol. 985, 37-59 (2013).

69. Barber, R. D., Harmer, D. W., Coleman, R. A. \& Clark, B. J. GAPDH as a housekeeping gene: analysis of GAPDH mRNA expression in a panel of 72 human tissues. Physiol. Genomics 21, 389-395 (2005).

70. Brasen, C., Esser, D., Rauch, B. \& Siebers, B. Carbohydrate metabolism in Archaea: current insights into unusual enzymes and pathways and their regulation. Microbiol Mol. Biol. Rev. 78, 89-175 (2014).

71. Siebers, B. \& Schonheit, P. Unusual pathways and enzymes of central carbohydrate metabolism in Archaea. Curr. Opin. Microbiol 8, 695-705 (2005).

72. Charron, C. et al. Crystallization and preliminary X-ray diffraction studies of D-glyceraldehyde-3-phosphate dehydrogenase from the hyperthermophilic archaeon Methanothermus fervidus. Acta Crystallogr D. Biol. Crystallogr 55, 1353-1355 (1999).

73. Heider, J., Ma, K. \& Adams, M. W. Purification, characterization, and metabolic function of tungsten-containing aldehyde ferredoxin oxidoreductase from the hyperthermophilic and proteolytic archaeon Thermococcus strain ES-1. J. Bacteriol. 177, 4757-4764 (1995).

74. Mukund, S. \& Adams, M. W. The novel tungsten-iron-sulfur protein of the hyperthermophilic archaebacterium, Pyrococcus furiosus, is an aldehyde ferredoxin oxidoreductase. Evidence for its participation in a unique glycolytic pathway. J. Biol. Chem. 266, 14208-14216 (1991).

75. Matsubara, K., Yokooji, Y., Atomi, H. \& Imanaka, T. Biochemical and genetic characterization of the three metabolic routes in Thermococcus kodakarensis linking glyceraldehyde 3-phosphate and 3-phosphoglycerate. Mol. Microbiol 81, 1300-1312 (2011).

76. Ettema, T. J., Ahmed, H., Geerling, A. C., van der Oost, J. \& Siebers, B. The non-phosphorylating glyceraldehyde-3-phosphate dehydrogenase (GAPN) of Sulfolobus solfataricus: a key-enzyme of the semi-phosphorylative branch of the Entner-Doudoroff pathway. Extremophiles 12, 75-88 (2008).

77. Brunner, N. A., Brinkmann, H., Siebers, B. \& Hensel, R. NAD+-dependent glyceraldehyde-3-phosphate dehydrogenase from Thermoproteus tenax. The first identified archaeal member of the aldehyde dehydrogenase superfamily is a glycolytic enzyme with unusual regulatory properties. J. Biol. Chem. 273, 6149-6156 (1998).

78. van der Oost, J. et al. The ferredoxin-dependent conversion of glyceraldehyde3 -phosphate in the hyperthermophilic archaeon Pyrococcus furiosus represents a novel site of glycolytic regulation. J. Biol. Chem. 273, 28149-28154 (1998)

79. Zwickl, P., Fabry, S., Bogedain, C., Haas, A. \& Hensel, R. Glyceraldehyde-3phosphate dehydrogenase from the hyperthermophilic archaebacterium Pyrococcus woesei: characterization of the enzyme, cloning and sequencing of the gene, and expression in Escherichia coli. J. Bacteriol. 172, 4329-4338 (1990).

80. Sakuraba, H. et al. Sequential aldol condensation catalyzed by hyperthermophilic 2-deoxy-d-ribose-5-phosphate aldolase. Appl Environ. Microbiol 73, 7427-7434 (2007). 
81. Sakuraba, H. et al. The first crystal structure of archaeal aldolase. Unique tetrameric structure of 2-deoxy-d-ribose-5-phosphate aldolase from the hyperthermophilic archaea Aeropyrum pernix. J. Biol. Chem. 278, 10799-10806 (2003).

82. Rashid, N., Imanaka, H., Fukui, T., Atomi, H. \& Imanaka, T. Presence of a novel phosphopentomutase and a 2-deoxyribose 5-phosphate aldolase reveals a metabolic link between pentoses and central carbon metabolism in the hyperthermophilic archaeon Thermococcus kodakaraensis. J. Bacteriol. 186, 4185-4191 (2004).

83. Lomax, M. S. \& Greenberg, G. R. Characteristics of the deo operon: role in thymine utilization and sensitivity to deoxyribonucleosides. J. Bacteriol. 96, 501-514 (1968).

84. Jia, B. et al. Proteome profiling of heat, oxidative, and salt stress responses in Thermococcus kodakarensis KOD1. Front Microbiol 6, 605 (2015).

85. Orita, I. et al. The ribulose monophosphate pathway substitutes for the missing pentose phosphate pathway in the archaeon Thermococcus kodakaraensis. J. Bacteriol. 188, 4698-4704 (2006).

86. Salleron, L. et al. DERA is the human deoxyribose phosphate aldolase and is involved in stress response. Biochim Biophys. Acta 1843, 2913-2925 (2014).

87. Niforou, K., Cheimonidou, C. \& Trougakos, I. P. Molecular chaperones and proteostasis regulation during redox imbalance. Redox Biol. 2, 323-332 (2014).

88. Burston, S. G. \& Clarke, A. R. Molecular chaperones: physical and mechanistic properties. Essays Biochem 29, 125-136 (1995).

89. De Oliveira, D. M. P. et al. Antimicrobial Resistance in ESKAPE Pathogens. Clin Microbiol Rev. 33, https://doi.org/10.1128/CMR.00181-19 (2020).

90. Saxena, S., Spaink, H. P. \& Forn-Cuni, G. Drug resistance in nontuberculous mycobacteria: mechanisms and models. Biology (Basel) 10, https://doi.org/ 10.3390/biology10020096 (2021).

91. Kanabalan, R. D. et al. Human tuberculosis and Mycobacterium tuberculosis complex: a review on genetic diversity, pathogenesis and omics approaches in host biomarkers discovery. Microbiol Res. 246, 126674 (2021).

92. Chomkatekaew, C., Boonklang, P., Sangphukieo, A. \& Chewapreecha, C. An evolutionary arms race between burkholderia pseudomallei and host immune system: what do we know? Front. Microbiol. 11, 612568 (2020).

93. Jose, R. J., Periselneris, J. N. \& Brown, J. S. Opportunistic bacterial, viral and fungal infections of the lung. Med. (Abingdon) 48, 366-372 (2020).

94. Bowman, J. A. \& Utter, G. H. Evolving strategies to manage Clostridium difficile colitis. J. Gastrointest. Surg. 24, 484-491 (2020).

95. Harvey, P. C. et al. Salmonella enterica serovar typhimurium colonizing the lumen of the chicken intestine grows slowly and upregulates a unique set of virulence and metabolism genes. Infect. Immun. 79, 4105-4121 (2011).

96. Klumpp, J. \& Fuchs, T. M. Identification of novel genes in genomic islands that contribute to Salmonella typhimurium replication in macrophages. Microbiol. (Read.) 153, 1207-1220 (2007).

97. Thiennimitr, P. et al. Intestinal inflammation allows Salmonella to use ethanolamine to compete with the microbiota. Proc. Natl Acad. Sci. USA 108, 17480-17485 (2011).

98. Srikumar, S. \& Fuchs, T. M. Ethanolamine utilization contributes to proliferation of Salmonella enterica serovar Typhimurium in food and in nematodes. Appl Environ. Microbiol 77, 281-290 (2011).

99. Pitts, A. C., Tuck, L. R., Faulds-Pain, A., Lewis, R. J. \& Marles-Wright, J. Structural insight into the Clostridium difficile ethanolamine utilisation microcompartment. PLoS ONE 7, e48360 (2012).

100. Maadani, A., Fox, K. A., Mylonakis, E. \& Garsin, D. A. Enterococcus faecalis mutations affecting virulence in the Caenorhabditis elegans model host. Infect. Immun. 75, 2634-2637 (2007).

101. Post, S. J., Shapiro, J. A. \& Wuest, W. M. Connecting iron acquisition and biofilm formation in the ESKAPE pathogens as a strategy for combatting antibiotic resistance. Medchemcomm 10, 505-512 (2019).

102. Soding, J. Protein homology detection by HMM-HMM comparison. Bioinformatics 21, 951-960 (2005).

103. Boto, L. Horizontal gene transfer in evolution: facts and challenges. Proc. Biol. Sci. 277, 819-827 (2010).

104. Kanhere, A. \& Vingron, M. Horizontal Gene Transfers in prokaryotes show differential preferences for metabolic and translational genes. BMC Evol. Biol. 9, 9 (2009).

105. Krupovic, M. \& Koonin, E. V. Multiple origins of viral capsid proteins from cellular ancestors. Proc. Natl Acad. Sci. USA 114, E2401-E2410 (2017).

106. Holm, L. DALI and the persistence of protein shape. Protein Sci. 29, 128-140 (2020).

107. Forterre, P. The origin of viruses and their possible roles in major evolutionary transitions. Virus Res. 117, 5-16 (2006).

108. Pastuzyn, E. D. et al. The neuronal gene Arc encodes a repurposed retrotransposon Gag protein that mediates intercellular RNA transfer. Cell 172, 275-288 e218 (2018).
109. Gerlt, J. A. et al. Enzyme function initiative-enzyme similarity tool (EFI-EST): a web tool for generating protein sequence similarity networks. Biochim Biophys. Acta 1854, 1019-1037 (2015).

110. Gerlt, J. A. Genomic enzymology: web tools for leveraging protein family sequence-function space and genome context to discover novel functions. Biochemistry 56, 4293-4308 (2017).

111. UniProt, C. UniProt: the universal protein knowledgebase in 2021. Nucleic Acids Res. 49, D480-D489 (2021).

112. Mistry, J. et al. Pfam: The protein families database in 2021. Nucleic Acids Res. 49, D412-D419 (2021).

113. Nichols, R. J. et al. Discovery and characterization of a novel family of prokaryotic nanocompartments involved in sulfur metabolism. bioRxiv, 2020.2005.2024.113720, https://doi.org/10.1101/2020.05.24.113720 (2020).

114. Eddy, S. R. Accelerated Profile HMM Searches. PLoS Comput Biol. 7, e1002195 (2011).

115. Sievers, F. et al. Fast, scalable generation of high-quality protein multiple sequence alignments using Clustal Omega. Mol. Syst. Biol. 7, 539 (2011).

116. Zimmermann, L. et al. A completely reimplemented MPI Bioinformatics toolkit with a new HHpred Server at its Core. J. Mol. Biol. 430, 2237-2243 (2018).

117. Gabler, F. et al. Protein sequence analysis using the MPI bioinformatics toolkit. Curr. Protoc. Bioinforma. 72, e108 (2020).

118. Hug, L. A. et al. A new view of the tree of life. Nat. Microbiol 1, 16048 (2016)

119. Letunic, I. \& Bork, P. Interactive Tree Of Life (iTOL) v4: recent updates and new developments. Nucleic Acids Res. 47, W256-W259 (2019)

120. Lang, A. S. \& Beatty, J. T. Importance of widespread gene transfer agent genes in alpha-proteobacteria. Trends Microbiol 15, 54-62 (2007).

121. Lemoine, F. et al. NGPhylogeny.fr: new generation phylogenetic services for non-specialists. Nucleic Acids Res. 47, W260-W265 (2019).

122. Katoh, K. \& Standley, D. M. MAFFT multiple sequence alignment software version 7: improvements in performance and usability. Mol. Biol. Evol. 30, 772-780 (2013).

123. Criscuolo, A. \& Gribaldo, S. BMGE (Block Mapping and Gathering with Entropy): a new software for selection of phylogenetic informative regions from multiple sequence alignments. BMC Evol. Biol. 10, 210 (2010).

124. Lefort, V., Longueville, J. E. \& Gascuel, O. SMS: smart model selection in PhyML. Mol. Biol. Evol. 34, 2422-2424 (2017)

125. Dickschat, J. S. Bacterial terpene cyclases. Nat. Prod. Rep. 33, 87-110 (2016)

126. Zallot, R., Oberg, N. O. \& Gerlt, J. A. 'Democratized' genomic enzymology web tools for functional assignment. Curr. Opin. Chem. Biol. 47, 77-85 (2018)

127. Shannon, P. et al. Cytoscape: a software environment for integrated models of biomolecular interaction networks. Genome Res. 13, 2498-2504 (2003).

128. Pettersen, E. F. et al. UCSF Chimera-a visualization system for exploratory research and analysis. J. Comput Chem. 25, 1605-1612 (2004).

129. Goddard, T. D. et al. UCSF ChimeraX: Meeting modern challenges in visualization and analysis. Protein Sci. 27, 14-25 (2018).

130. Jones, D. T. \& Cozzetto, D. DISOPRED3: precise disordered region predictions with annotated protein-binding activity. Bioinformatics $\mathbf{3 1}$, 857-863 (2015)

131. Sekiguchi, Y. et al. First genomic insights into members of a candidate bacterial phylum responsible for wastewater bulking. PeerJ 3, e740 (2015).

\section{Acknowledgements}

We gratefully acknowledge funding from the NIH (R35GM133325).

\section{Author contributions}

M.P.A and T.W.G designed the study, carried out computational analyses, and wrote the paper.

\section{Competing interests}

The authors declare no competing interests.

\section{Additional information}

Supplementary information The online version contains supplementary material available at https://doi.org/10.1038/s41467-021-25071-y.

Correspondence and requests for materials should be addressed to T.W.G.

Peer review information Nature Communications thanks the anonymous reviewers for their contribution to the peer review of this work.

Reprints and permission information is available at http://www.nature.com/reprints

Publisher's note Springer Nature remains neutral with regard to jurisdictional claims in published maps and institutional affiliations. 
(c) (i) Open Access This article is licensed under a Creative Commons Attribution 4.0 International License, which permits use, sharing, adaptation, distribution and reproduction in any medium or format, as long as you give appropriate credit to the original author(s) and the source, provide a link to the Creative Commons license, and indicate if changes were made. The images or other third party material in this article are included in the article's Creative Commons license, unless indicated otherwise in a credit line to the material. If material is not included in the article's Creative Commons license and your intended use is not permitted by statutory regulation or exceeds the permitted use, you will need to obtain permission directly from the copyright holder. To view a copy of this license, visit http://creativecommons.org/ licenses/by/4.0/.

(C) The Author(s) 2021 